\title{
Multidimensional hydrogen tunneling dynamics in the ground vibrational state of the ammonia dimer
}

\author{
Jennifer G. Loeser, C. A. Schmuttenmaer, ${ }^{\text {a) }}$ R. C. Cohen, ${ }^{\text {b) }}$ M. J. Elrod, D. W. Steyert, ${ }^{\text {c) }}$ \\ and R. J. Saykally \\ Department of Chemistry, University of California, Berkeley, California 94720 \\ R. E. Bumgarner ${ }^{\text {f) }}$ and G. A. Blake \\ Division of Geological and Planetary Sciences, California Institute of Technology, Pasadena, \\ California 91125
}

(Received 20 March 1992; accepted 27 April 1992)

\begin{abstract}
We have measured and assigned more than 800 new far-infrared absorption lines and 12 new microwave absorption lines of the ammonia dimer. Our data are analyzed in combination with all previously measured far-infrared and microwave spectra for this cluster. The vibration-rotation-tunneling (VRT) states of the ammonia dimer connected by electricdipole-allowed transitions are separated into three groups that correspond to different combinations of monomer rotational states: $A+A$ states (states formed from the combination of two ammonia monomers in $A$ states), $A+E$ states, and $E+E$ states. We present complete experimentally determined energy-level diagrams for the $K_{a}=0$ and $K_{a}=1$ levels of each group in the ground vibrational state of this complex. From these, we deduce that the appropriate molecular symmetry group for the ammonia dimer is $G_{144}$. This, in turn, implies that three kinds of tunneling motions are feasible for the ammonia dimer: interchange of the "donor" and "acceptor" roles of the monomers, internal rotation of the monomers about their $C_{3}$ symmetry axes, and quite unexpectedly, "umbrella" inversion tunneling. In the $K_{a}=0 A+E$ and $E+E$ states, the measured umbrella inversion tunneling splittings range from 1.1 to $3.3 \mathrm{GHz}$. In $K_{a}=1$, these inversion splittings between two sets of $E+E$ states are 48 and $9 \mathrm{MHz}$, while all others are completely quenched. Another surprise, in light of previous analyses of tunneling in the ammonia dimer, is our discovery that the interchange tunneling splittings are large. In the $A+A$ and $E+E$ states, they are 16.1 and $19.3 \mathrm{~cm}^{-1}$, respectively. In the $A+E$ states, the measured $20.5 \mathrm{~cm}^{-1}$ splitting can result from a difference in "donor" and "acceptor" internal rotation frequencies that is increased by interchange tunneling. We rule out the possibility that the upper state of the observed far-infrared subbands is the very-low-frequency out-of-plane intermolecular vibration predicted in several theoretical studies [C. E. Dykstra and L. Andrews, J. Chem. Phys. 92, 6043 (1990); M. J. Frisch, J. E. Del Bene, J. S. Binkley, and H. F. Schaefer III, ibid. 84, 2279 (1986)]. In their structure determination, Nelson et al. assumed that monomer umbrella inversion tunneling was completely quenched and that "donor-acceptor" interchange tunneling was nearly quenched in the ammonia dimer [D. D. Nelson, G. T. Fraser, and W. Klemperer, J. Chem. Phys. 83, 6201 (1985); D. D. Nelson, W. Klemperer, G. T. Fraser, F. J. Lovas, and R. D. Suenram, ibid. 87, 6364 (1987)]. Our experimental results, considered together with the results of sixdimensional calculations of the VRT dynamics presented by van Bladel et al. in the accompanying paper [J. Chem. Phys. 97, 4750 (1992)], make it unlikely that the structure proposed by Nelson et al. for the ammonia dimer is the equilibrium structure.
\end{abstract}

\section{INTRODUCTION}

Over the past decade, a considerable variety of hydrogen-bonded dimers has been investigated by highresolution spectroscopic methods ${ }^{1-3}$ and in parallel by high-level $a b$ initio techniques. ${ }^{4}$ The goal of this work has

"Present address: Department of Chemistry, University of Rochester, Rochester, NY 14267.

b) Present address: Department of Chemistry, Harvard University, Cambridge, MA 02138 .

c) Present address: Department of Chemistry, Colgate University, Hamilton, NY 13346.

d) Present address: Division of Biology, California Institute of Technology, Pasadena, CA 91125 . been (1) to establish a predictive model for the dimer equilibrium structures and ultimately for the internal dynamics of small hydrogen-bonded clusters, and (2) to connect this detailed molecular knowledge with the behavior of the constituents in condensed phases. For the most part, good agreement has been obtained between theory and experiment for these prototypical systems. The most well-known exception is the singular case of the ammonia dimer.

For 23 years, since the matrix studies of Pimentel, Bulanin, and van Thiel, ${ }^{5}$ chemists had generally assumed that the minimum of the ammonia dimer intermolecular potential-energy surface corresponded to a linearly hydrogen-bonded geometry, ${ }^{6}$ similar to that determined for the water dimer. ${ }^{7}$ Figure 1 , which shows a set of intermo- 

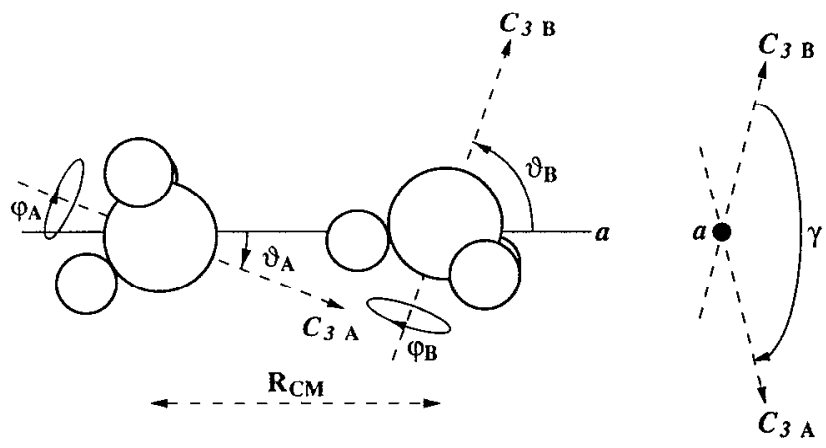

FIG. 1. The six intermolecular coordinates of the ammonia dimer. For clarity, we show the coordinate system used in the following paper (Ref. 22). Most important in our discussion are the angles $\theta_{A}$ and $\theta_{B}$ between the ammonia monomer symmetry axes and the $a$ inertial axis of the dimer. These angles define the roles of the inequivalent ammonia monomers as "acceptor" and "donor." Donor-acceptor interchange tunneling, a large-amplitude motion which reverses these roles is shown to be a very facile motion in the ammonia dimer. The angles $\phi_{A}$ and $\phi_{B}$ describe internal rotations of the monomers about their symmetry axes, labeled $C_{3 A}$ and $C_{3 B}$. Internal rotation also occurs readily in the ammonia dimer. The dihedral angle $\gamma$ is the angle between the two planes that each contain one monomer symmetry axis and the a inertial axis of the complex. In this paper, we assume that the ammonia dimer contains a plane of symmetry; that is, we assume that $\gamma=180^{\circ}$ (or $0^{\circ}$ ).

lecular coordinates for the ammonia dimer, illustrates one possible hydrogen-bonded configuration. However, Nelson, Fraser, and Klemperer's analysis of their molecularbeam electric resonance (MBER) rotational spectra ${ }^{8,9}$ indicated that the vibrationally averaged structure of the ammonia dimer was not a hydrogen-bonded one. Rather, they argued that while the complex was clearly asymmetric, the equilibrium structure was closer to being cyclic and centrosymmetric than linearly hydrogen bonded. In the coordinate system of Fig. 1, this proposed structure has $\theta_{A}=48.6^{\circ}$ and $\theta_{B}=115.5^{\circ}$, while $\phi_{A}, \phi_{B}$, and $\gamma$ are not determined. This unexpected result was determined in a straightforward way from the observed $J=1 \leftarrow 0$ and $J=2 \leftarrow 1$ pure rotational transitions in two distinct "vibrational" states of the ammonia dimer. The measured ${ }^{14} \mathrm{~N}$ quadrupole coupling constants $e q Q_{a a}$ for each ammonia monomer, together with the $\mu_{a}$ dipole moment component of the complex, were inverted to determine the two angles $\theta_{A}$ and $\theta_{B}$ between the ammonia symmetry axes and the a inertial axis of the dimer.

Subsequently, Nelson and Klemperer ${ }^{10}$ presented an elegant group-theoretical description of some of the possible large-amplitude hydrogen tunneling motions that might occur within the ammonia dimer. We will discuss the group-theoretical analysis of Nelson and Klemperer in more detail later in this paper, since their analysis provides a starting point for our own. Nelson and Klemperer considered the possible effects of a "donor-acceptor" interchange tunneling motion, similar to that found in the $(\mathrm{HF})_{2},{ }^{11,12}(\mathrm{HCl})_{2},{ }^{13}$ and $\left(\mathrm{H}_{2} \mathrm{O}\right)_{2},{ }^{14,15}$ along with the possible effects of the internal rotation of the monomers about their symmetry axes on the rovibrational states of the ammonia dimer. They assumed that the umbrella inversion of the monomers would be totally quenched by anisotropy in the ammonia dimer potential. Under these assumptions, two sets of pure rotational transitions are predicted to occur within the lowest $K_{a}=0$ manifold of the ammonia dimer. The two tunneling states that give rise to these transitions are shown to be separated by the difference in internal rotation frequencies of the "donor" and "acceptor," and in second order, their separation can be increased by interchange tunneling. Applying their group-theoretical model to the MBER data, Nelson et al. ${ }^{9}$ argued that the donor-acceptor interchange tunneling frequency was very small in the ammonia dimer because the two tunneling states accessed in their spectra appeared to lie very close in energy. In addition, since they measured different ${ }^{14} \mathrm{~N}$ quadrupole coupling constants for each monomer, they deduced that the donor-acceptor interchange tunneling had no pronounced averaging effect on the angles derived, in the usual way, from these measured ${ }^{14} \mathrm{~N}$ quadrupole coupling constants. Furthermore, careful isotopic substitution experiments revealed that similarly determined structures for a variety of ammonia dimer isotopomers were nearly identical. Nelson et al. inferred from these results that the equilibrium structure of the ammonia dimer was not very different from the vibrationally averaged structure that they determined. It is this claim that defines the apparent discrepancy between theory and experiment that has existed since the appearance of the MBER work.

Several extensive $a b$ initio theoretical investigations ${ }^{16-20}$ have followed the work of Klemperer group. In these studies, vibrational averaging, both from the tunneling motions, and from the floppy intermolecular vibrations of the ammonia dimer complex, is cited as the cause of the difference between the predicted equilibrium structure and the experimental results. Indeed, the intermolecular potential-energy surface of the ammonia dimer is generally found to be rather flat in some angular coordinates. Both Frisch et al. ${ }^{16}$ and Hassett, Marsden, and Smith ${ }^{20}$ found a linearly hydrogen-bonded equilibrium structure, and a cyclic, centrosymmetric transition state for the interchange motion between two such potential minima that was only $0.1-0.2 \mathrm{kcal} / \mathrm{mol}$ higher in energy. Both of these groups also found that the harmonic zero-point energy of the intermolecular vibration in the interchange tunneling coordinate was approximately equal to the interchange barrier height.

The first high-resolution experiment to probe the other types of ammonia dimer tunneling states, which do not support pure rotational spectra, was performed by $\mathrm{Ha}$ venith et al., ${ }^{21}$ who discovered ammonia dimer spectra between 21 and $28 \mathrm{~cm}^{-1}$, using tunable far-infrared laser spectroscopy. Although this work dramatically increased the number of known ammonia dimer vibration-rotationtunneling (VRT) states from 2 to 12 , none of the farinfrared (FIR) transitions shared common upper or lower states. Thus, relatively little information on the groundstate tunneling energetics or dynamics could be extracted from this subset of the FIR spectrum. The results of Havenith et al. were interpreted as support for the conclusions of Nelson et al., since no inconsistencies with the microwave work were revealed at that time. 
In this paper, we present a far more complete experimental characterization of the ground vibrational state of the ammonia dimer than has been possible before. From our extensive new data set, we are able to show that the appropriate molecular symmetry group for the ammonia dimer complex is $G_{144}$. Thus, the donor-acceptor interchange tunneling, internal rotation of the $\mathrm{NH}_{3}$ monomers about their $C_{3}$ symmetry axes, and monomer "umbrella" inversion tunneling are all feasible. We have precisely measured these interchange and inversion tunneling frequencies. Furthermore, we find that the two VRT states sampled by the pure rotational spectra are separated by the inversion tunneling frequency, not the interchange tunneling frequency. Indeed, the assumptions of Nelson et $a l^{8-10}$ that umbrella inversion tunneling does not occur and that the interchange tunneling frequency is therefore very small are clearly invalid. Quite the opposite tunneling scheme is appropriate for this complex: we find that the interchange tunneling frequency is likely to be the largest of all hydrogen tunneling frequencies in the ammonia dimer.

The first explicit calculation of the VRT states on one of the $a b$ initio ammonia dimer surfaces ${ }^{19}$ is described by van Bladel et al. ${ }^{22}$ in the accompanying paper. The qualitative picture obtained from the wave functions and relative energies thus computed has led these authors to anticipate the results found experimentally in our own work. More specifically, based on their calculations, they suggest assignments of the previously observed subbands near 483 and $614 \mathrm{GHz}$ which are the same as the assignments that we have deduced by careful consideration of our data alone. Although it was not included in their calculations, van Bladel et al. also suggest that the ammonia monomer umbrella inversion tunneling motion may be occurring in the ammonia dimer complex. This result is unambiguously proven in our own analysis presented below. van Bladel $e t$ al. confirm the hypothesis of Nelson et al. that the wave functions of tunneling states that can be sampled by pure rotational transitions are to some extent "localized" with respect to interchange tunneling, and that one can therefore expect to measure two different ${ }^{14} \mathrm{~N}$ quadrupole coupling constants for the two different ammonia monomers in such states. However, they also show that the assumption of Nelson, Fraser, and Klemperer ${ }^{8}$ that the quadrupole coupling constants, measures of $\left\langle P_{2}\left(\cos \theta_{A}\right)\right\rangle$ and $\left\langle P_{2}\left(\cos \theta_{B}\right)\right\rangle$, can be used in conjunction with the dipole moment, a measure of $\left\langle P_{1}\left(\cos \theta_{A}\right)\right\rangle$ and $\left\langle P_{1}\left(\cos \theta_{B}\right)\right\rangle$, to deduce structural information may not be appropriate.

We emphasize that, even though some of our results and some of the results of van Bladel et al. ${ }^{22}$ are strikingly similar, each group performed its own analysis and drew corresponding logical conclusions before learning of the other's results. This agreement constitutes additional strong support for the independent analyses presented in both papers.

Here, we present three separate spectroscopically determined energy-level diagrams for the $K_{a}=0$ and $K_{a}=1$ levels of the ammonia dimer, corresponding to three different kinds of internal rotor states. These incorporate our new data set, the spectra previously reported by Havenith et al. ${ }^{21}$ and the spectra reported by Zwart. ${ }^{23}$ First, we describe our rotational analysis of these data and the observed Coriolis interactions. Then we discuss in more detail the physical and group-theoretical nature of the tunneling states of the ammonia dimer. Finally, we present our tunneling and vibrational assignment of the observed farinfrared and microwave transitions.

\section{EXPERIMENT}

The Berkeley tunable far-infrared laser spectrometers have been described previously. ${ }^{24}$ Since the work of Havenith et al. ${ }^{21}$ on the $\left(\mathrm{NH}_{3}\right)_{2}$ spectrum, we have added the following improvements: (1) we have optimized production of $\left(\mathrm{NH}_{3}\right)_{2}$ by varying the concentration of ammonia in the argon carrier gas using mass flow controllers, (2) we have increased the effective sample path length of the tunable far-infrared laser sidebands (fixed-frequency farinfrared laser radiation mixed with tunable microwave radiation) through the planar jet expansion by multipassing ${ }^{25}$ (usually 10 times) the sidebands in front of a longer $(10 \mathrm{~cm})$ slit nozzle, ${ }^{26}(3)$ we have increased the far-infrared transmission to the detector by purging the entire beam path with dry nitrogen, and (4) we have increased our "routine" (but not "ultimate") detection sensitivity by subtracting the base-line variation due to microwave transmission resonances, and also the resonances of the sidebands inside the far-infrared laser cavity, from every scan. We have found it fruitful to rescan much of the region originally covered by Havenith et al. ${ }^{21}$ The frequencies and lasing mediums (given in parentheses) of the fixed-frequency far-infrared lasers used in the present study were $527.9260 \mathrm{GHz}$ (DCOOD), $584.3882 \mathrm{GHz}$ $(\mathrm{HCOOH}), 639.1846 \mathrm{GHz}\left(\mathrm{CH}_{3} \mathrm{OH}\right), 692.9513 \mathrm{GHz}$ $(\mathrm{HCOOH}), 716.1568 \mathrm{GHz}(\mathrm{HCOOH}), 761.6083 \mathrm{GHz}$ $(\mathrm{HCOOH}), 768.8820 \mathrm{GHz}$ (DCOOD), $787.7555 \mathrm{GHz}$ (DCOOD), $850.4118 \mathrm{GHz}\left(\mathrm{CH}_{3} \mathrm{OD}\right), 939.4940 \mathrm{GHz}$ $\left(\mathrm{CH}_{3} \mathrm{OD}\right), 980.5916 \mathrm{GHz}\left(\mathrm{CH}_{3} \mathrm{OD}\right), 1016.8972 \mathrm{GHz}$ $\left(\mathrm{CH}_{3} \mathrm{OD}\right), 1042.1504 \mathrm{GHz}\left(\mathrm{CH}_{2} \mathrm{~F}_{2}\right), 1101.1594 \mathrm{GHz}$ $\left(\mathrm{CH}_{2} \mathrm{DOH}\right), 1110.3199 \mathrm{GHz}\left(\mathrm{CH}_{2} \mathrm{~F}_{2}\right), 1145.4302 \mathrm{GHz}$ $\left(\mathrm{CH}_{2} \mathrm{~F}_{2}\right)$, and $1193.7273 \mathrm{GHz}\left(\mathrm{CH}_{3} \mathrm{OH}\right) .{ }^{27}$ The $584-768$ $\mathrm{GHz}$ lasers were scanned at the highest sensitivity.

Our group has measured a total of nearly 4000 farinfrared absorption lines due to $\mathrm{Ar}-\mathrm{NH}_{3},\left(\mathrm{NH}_{3}\right)_{2}$, and other as-yet unanalyzed $\mathrm{Ar}_{n}-\left(\mathrm{NH}_{3}\right)_{m}$ clusters. Over 950 of these are assigned to $\left(\mathrm{NH}_{3}\right)_{2}$. The signal-to-noise ratio observed on the strongest $\left(\mathrm{NH}_{3}\right)_{2}$ absorption approaches $10000: 1$, while the weakest assigned lines have a signal-tonoise ratio of only 3:1. (For comparison, Havenith et al. ${ }^{21}$ report a signal-to-noise ratio of 100:1 for the strongest lines they were able to assign to the ammonia dimer.)

The Caltech microwave direct absorption spectrometer has also been described previously. ${ }^{28}$ Continuous searches were conducted in the regions $29.000-33.140 \mathrm{GHz}$, $40.800-57.735 \mathrm{GHz}$, and $60.900-62.286 \mathrm{GHz}$. Typical scanning conditions allowed observation of the $\left(\mathrm{NH}_{3}\right)_{2}$ pure rotational spectrum, the " $G_{\alpha}$ " and " $G_{\beta}$ " states discovered by the Klemperer group, ${ }^{8,9}$ at a signal-to-noise ratio of several hundred to one. 
Most of the microwave lines that we observed were part of the well-studied microwave spectrum of the $E$ states of $\mathrm{Ar}-\mathrm{NH}_{3}{ }^{29}$ the pure rotational progressions of $\left(\mathrm{NH}_{3}\right)_{2}{ }^{8,9}$ as well as a few high- $J$ lines in the pure inversion spectrum of the ammonia monomer. ${ }^{30}$ We have measured 20 microwave absorption lines due to other species or series, and assign 12 of these to a new rotationtunneling subband of the ammonia dimer, as described later in this paper. The measured intensities of these new lines were about 2 orders of magnitude less that the observed intensities of the $K_{a}=0$ pure rotational transitions.

\section{ANALYSIS}

\section{A. Rotational analysis}

A stick spectrum indicating the positions and estimated relative intensities of all the far-infrared absorption lines currently assigned to the ammonia dimer is presented in Fig. 2(a). (The intensities shown are calculated for $5 \mathrm{~K}$, with adjustments made for obvious intensity perturbations.) Figure 2(b) is a chart indicating the new observed microwave transitions. (The symmetry assignments indicated in this chart are discussed later in this paper.) The relative energies of the vibration-rotation-tunneling (VRT) states are diagrammed in Figs. 3(a), 3(b), and 3 (c), and the subband origins of the electric dipole transitions that connect the VRT states shown in these figures are listed in Table II. [The complete list of all the observed transitions (Table I) can be obtained from PAPS. ${ }^{31}$ ] These states are separated into three groups because of internal rotation of the ammonia monomers within the complex. For clarity, we label these groups by the symmetry types of the constituent ammonia monomer states: $A+A, A+E$, and $E+E$. The relative energies of all states within each group are now completely determined for $K_{a}=0$ and $K_{a}=1$, and all of the transitions between states in the same group are fit simultaneously. The rotational parameters determined in each fit are listed in Table III and the lowest resulting calculated VRT energy levels (up to $J=5$ ) are listed in Table IV. (The frequencies of all the allowed low$J$ transitions can easily be calculated from the data in Tables IV to within $1 \mathrm{MHz}$.) One purpose of performing these fits is to quantitatively establish that the observed transitions have the common upper and lower states indicated in the energy-level diagrams. The simultaneous fits are also necessary to treat the strong Coriolis interations between certain $K_{a}=0$ levels and nearby $K_{a}=1$ levels with the same symmetry. Strong Coriolis perturbations occur in the $A+E$ states about $600 \mathrm{GHz}$ above the lowest $A+E$ state and even stronger Coriolis perturbations are present in the lowest $E+E$ states. The VRT subbands which involve these affected states cannot be fit without this full Coriolis analysis.

For clarity and convenience, we have arbitrarily numbered the vibration-tunneling (VT) manifolds in each of the three groups of states. This numbering is indicated in the energy-level diagrams and identifies each transition in the line lists. $K_{a} \neq 0$ manifolds have two numbers, and the smaller number labels lower-energy component of each $K$ -
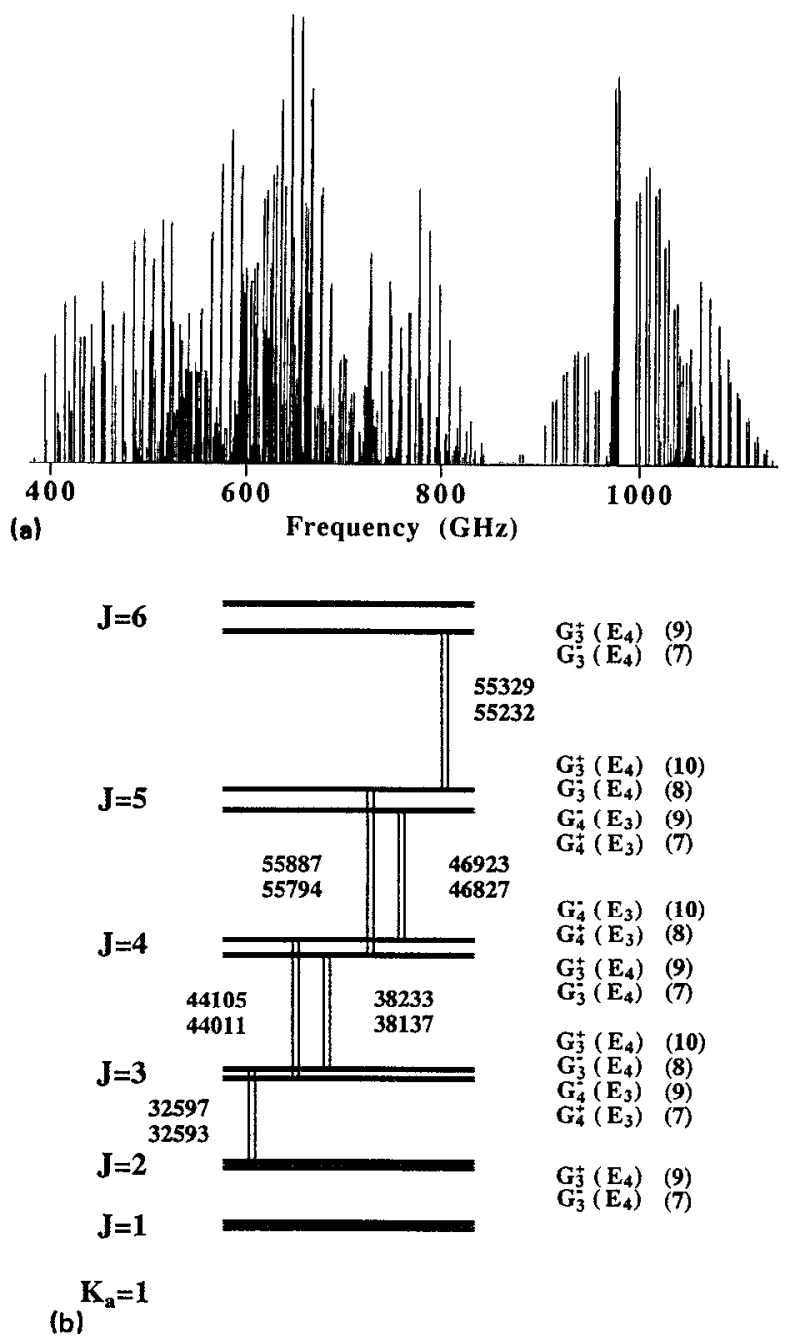

FIG. 2. (a) Stick spectrum of all the observed far-infrared transitions that have been assigned to the ammonia dimer between 13 and $38 \mathrm{~cm}^{-1}$. The transitions assigned by Havenith (Ref. 21) and by Zwart (Ref. 23) are included. These lines represent about one-fourth of all the absorptions we have measured in the $16-41 \mathrm{~cm}^{-1}$ frequency region in $\mathrm{Ar} / \mathrm{NH}_{3}$ expansions. Since these transitions were recorded over the course of several years under varying experimental conditions, this figure shows intensities calculated for a $5 \mathrm{~K}$ supersonic expansion, rather than experimentally observed signal strengths. In certain $\left|\Delta K_{a}\right|=1$ subbands that involve strongly perturbed $K_{a}=0$ or $K_{c}=1$ manifolds, the observed $R$ (or $P$ ) branch transitions are apparently an order of magnitude or more stronger than the weaker $P$ (or $R$ ) branch transitions. We have scaled the calculated intensities of these branches to approximately preserve this unexplained effect. (b) Rotation-tunneling energy-level diagram for two of the $K_{u}=1 E+E$ manifolds of the ammonia dimer. The 12 new microwave transitions are indicated. The small $48 \mathrm{MHz}$ splittings are the nearly quenched ammonia monomer umbrella inversion tunneling frequency. The asymmetry doubling, which is larger than the tunneling splitting, is especially large due to Coriolis coupling of the upper components of the asymmetry doublets to nearby $K_{a}=0$ states. The numbers (7), (8), (9), and $(10)$ are used for identification of these states in the text. The symmetry assignments are also discussed in the text.

type doublet. For each VT level $n$, the corresponding endover-end rotational states are fit to the phenomenological expressions:

$$
E=E_{n}+B_{n} J(J+1)-D_{n}[J(J+1)]^{2}+H_{n}[J(J+1)]^{3}+\cdots,
$$



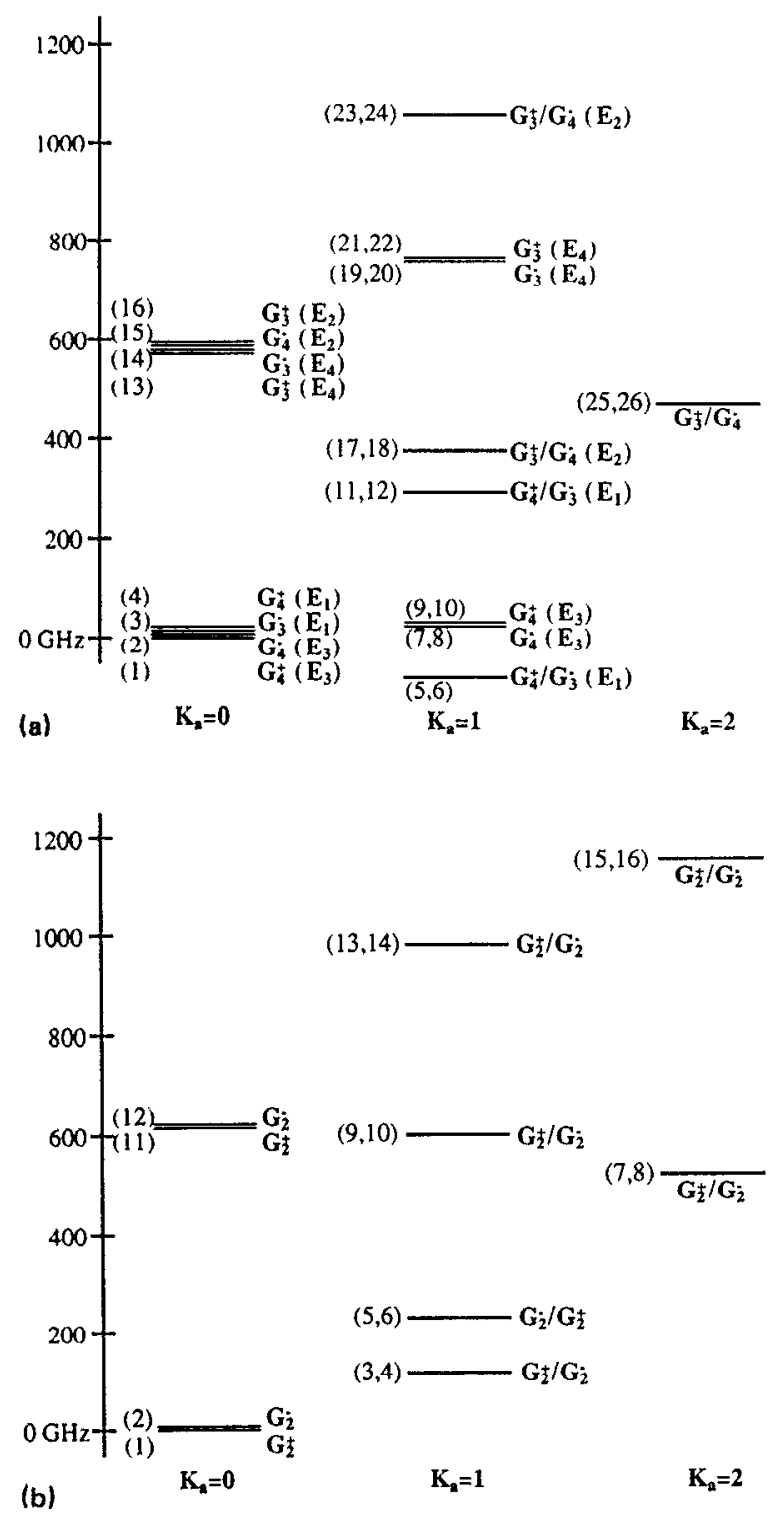

$$
\begin{aligned}
E^{ \pm}= & E_{n}+B_{n} J(J+1)-D_{n}[J(J+1)]^{2}+H_{n}[J(J+1)]^{3} \\
& +\cdots \pm\left[q B_{n} J(J+1)+q D_{n}[J(J+1)]^{2}+\cdots\right] .
\end{aligned}
$$

We use $E_{n}$ to refer to the $J=0$ rotational state of each VT level $n$. Since we treat levels with different $K_{a}$ values as separate "vibrations," $E_{n}$ for $K_{a} \neq 0$ levels is the energy of a fictitious rotational state. We use expression (1) for all $K_{a}=0$ manifolds and we use expression (2) for the $K_{a} \neq 0$ levels that appear to have a true "asymmetry" doubling in the absence of perturbations. Some of the $K_{a}=1$ states are split only by Coriolis interaction with nearby $K_{a}=0$ states. When this effect is strong, we use expression (1) for both the $K_{a}=0$ and $K_{a}=1$ manifolds and determine the Coriolis coupling constant as described below. The Coriolis interaction pushes the $K_{a}=0$ level and one of the initially degenerate $K_{a}=1$ levels apart. When this effect is weak, the Coriolis model still fits the data very well; however, the values of $B$ and the Coriolis coupling constants are totally correlated and also not very well determined. In these weakly interacting cases we choose to present fits in which

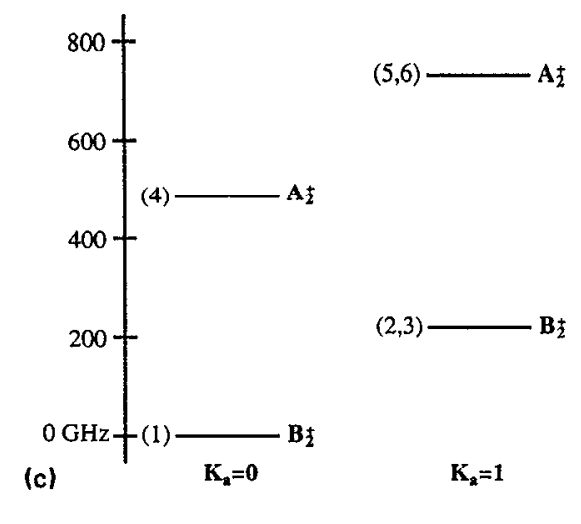

FIG. 3. (a) Complete energy-level diagram for the $E+E$ tunneling states of the ammonia dimer. The observed tunneling levels (horizontal lines) are labeled by the arbitrary numbers $(n)$ used for clarity in the text and are positioned according to the relative energies $E_{n}$ determined in our fit. Table II(a) lists all of the observed VRT subbands that connect these $E+E$ states. The indicated tunneling symmetries in $G_{144}$ are taken to be the symmetries of the even rotational states. The symmetries of the $G_{36}$ states that were split by the monomer inversion to give the $G_{144}$ states are shown in parentheses. Table IV (a) tabulates the assigned symmetries and relative energies of the lowest few rotational levels in each of the observed $E+E$ tunneling states. (b) Complete energy-level diagram for the $A+E$ tunneling states of the ammonia dimer. The observed tunneling levels (horizontal lines) are labeled by the arbitrary numbers $(n)$ used for clarity in the text and are positioned according to the relative energies $E_{n}$ determined in our fit. Table II(b) lists all of the observed VRT subbands that connect these $A+E$ states. The indicated tunneling symmetries in $G_{144}$ are taken to be the symmetries of the even rotational states. Table IV(b) tabulates the assigned symmetries and relative energies of the lowest few rotational levels in each of the observed $A+E$ tunneling states. (c) Complete energy-level diagram for the $A+A$ tunneling states of the ammonia dimer. The observed tunneling levels (horizontal lines) are labeled by the arbitrary numbers $(n)$ used for clarity in the text and are positioned according to the relative energies $E_{n}$ determined in our fit. Table II (c) lists all of the observed VRT subbands that connect these $A+A$ states. The indicated tunneling symmetries in $G_{144}$ are taken to be the symmetries of the even rotational states. Table IV(c) tabulates the assigned symmetries and relative energies of the lowest few rotational levels in each of the observed $A+A$ tunneling states.

we use expression (1) for the $K_{a}=0$ manifold and expression (2) for the $K_{a}=1$ manifold with no Coriolis coupling included. Still other $K_{a}=1$ states are split both by Coriolis and asymmetry doubling effects.

We find that the rotational states within some VT manifolds cannot be fit well using only $B$ and a small value of $D$ (less than $0.1 \mathrm{MHz}$ ) in expression (1) or (2). Usually these perturbed manifolds come in pairs of one $K_{a}=0 \mathrm{VT}$ level near one $K_{a}=1$ VT level, or one $K_{a}=1$ VT level near one $K_{a}=2$ VT level. The two levels in each pair have second- and third-order distortion constants with similar magnitudes but opposite signs. We model the interaction between such pairs of manifolds as Coriolis coupling. In such cases, we first check that each pair of interacting levels can be connected by two successive electric-dipoleallowed transitions. This assures that the states in question have the same symmetry. We then fit off-diagonal matrix elements of the form $c[J(J+1)]^{1 / 2}$ between the appropriate unperturbed VRT levels of the same $J$ and the same symmetry in $K_{a}=0$ and $K_{a}=1$. Matrix elements appropriate for coupling $K_{a}=1$ states to $K_{a}=2$ states are 
TABLE II. Band origins for observed transitions between (a) $E+E$, (b) $A+E$, and (c) $A+A$ states.

\begin{tabular}{|c|c|c|c|c|}
\hline $\begin{array}{l}\text { Tunneling } \\
\text { symmetry }\end{array}$ & $K_{a}^{\prime} \leftarrow K_{a}^{\prime}$ & $\left(n^{\prime}\right) \leftarrow\left(n^{\prime \prime}\right)$ & $\begin{array}{c}\text { Number } \\
\text { of } \\
\text { lines }\end{array}$ & $E_{n^{\prime}}-E_{n^{\prime \prime}}$ \\
\hline
\end{tabular}

(a)

$\begin{array}{lcccc}G_{4}^{-}\left(E_{3}\right)-G_{4}^{+}\left(E_{3}\right) & 1 \leftarrow 1 & (7,8)-(9,10) & 12 & 48.2 \mathrm{MHz} \\ G_{3}^{+} / G\left(G_{4}^{-}\left(E_{2}\right) \leftarrow G_{4}^{+} / G_{3}^{-}\left(E_{1}\right)\right. & 1 \leftarrow 1 & (17,18) \leftarrow(5,6) & 26 & 453.6 \mathrm{GHz} \\ G_{3}^{+}\left(E_{4}\right)-G_{4}^{+}\left(E_{3}\right) & 0 \leftarrow 1 & (13)-(9,10) & 19 & 548.7 \mathrm{GHz} \\ G_{3}^{-}\left(E_{4}\right)-G_{4}^{-}\left(E_{3}\right) & 0 \leftarrow 1 & (14) \leftarrow(7,8) & 18 & 551.0 \mathrm{GHz} \\ G_{3}^{+}\left(E_{2}\right) \leftarrow G_{4}^{+}\left(E_{3}\right) & 0 \leftarrow 1 & (16)-(9,10) & 15 & 554.7 \mathrm{GHz} \\ G_{3}^{+}\left(E_{4}\right)-G_{4}^{+}\left(E_{1}\right) & 0 \leftarrow 0 & (13) \leftarrow(4) & 14 & 575.5 \mathrm{GHz} \\ G_{3}^{+}\left(E_{4}\right)-G_{4}^{+}\left(E_{3}\right) & 0 \leftarrow 0 & (13) \leftarrow(1) & 23 & 577.9 \mathrm{GHz} \\ G_{3}^{-}\left(E_{4}\right)-G_{4}^{-}\left(E_{3}\right) & 0 \leftarrow 0 & (14) \leftarrow(2) & 21 & 579.0 \mathrm{GHz} \\ G_{4}^{-}\left(E_{2}\right) \leftarrow G_{3}^{-}\left(E_{1}\right) & 0 \leftarrow 0 & (15) \leftarrow(3) & 19 & 580.3 \mathrm{GHz} \\ G_{3}^{+}\left(E_{2}\right) \leftarrow G_{4}^{+}\left(E_{1}\right) & 0 \leftarrow 0 & (16) \leftarrow(4) & 23 & 581.4 \mathrm{GHz} \\ G_{3}^{+}\left(E_{2}\right)-G_{4}^{+}\left(E_{3}\right) & 0 \leftarrow 0 & (16) \leftarrow(1) & 17 & 583.8 \mathrm{GHz} \\ G_{3}^{+}\left(E_{4}\right) \leftarrow G_{4}^{+} / G_{3}^{-}\left(E_{1}\right) & 0 \leftarrow 1 & (13) \leftarrow(5,6) & 23 & 655.5 \mathrm{GHz} \\ G_{4}^{-}\left(E_{2}\right)-G_{4}^{+} / G_{3}^{-}\left(E_{1}\right) & 0 \leftarrow 1 & (15) \leftarrow(5,6) & 25 & 659.2 \mathrm{GHz} \\ G_{3}^{+}\left(E_{2}\right)-G_{4}^{+} / G_{3}^{-}\left(E_{1}\right) & 0 \leftarrow 1 & (16) \leftarrow(5,6) & 21 & 661.4 \mathrm{GHz} \\ G_{3}^{+}\left(E_{4}\right)-G_{4}^{+}\left(E_{3}\right) & 1 \leftarrow 1 & (21,22) \leftarrow(9,10) & 53 & 730.78 \mathrm{GHz} \\ G_{3}^{-}\left(E_{4}\right) \leftarrow G_{4}^{-}\left(E_{3}\right) & 1 \leftarrow 1 & (19,20) \leftarrow(7,8) & 53 & 730.82 \mathrm{GHz} \\ G_{3}^{+} / G_{4}^{-}(? ?)-G_{4}^{+} / G_{3}^{-}\left(E_{1}\right) & 2 \leftarrow 1 & (25,26) \leftarrow(5,6) & 24 & 746.4 \mathrm{GHz} \\ G_{3}^{+}\left(E_{4}\right) \leftarrow G_{4}^{+}\left(E_{1}\right) & 1 \leftarrow 0 & (21,22) \leftarrow(4) & 4 & 757.6 \mathrm{GHz} \\ G_{3}^{-}\left(E_{4}\right)-G_{4}^{-}\left(E_{3}\right) & 1 \leftarrow 0 & (19,20) \leftarrow(2) & 18 & 758.8 \mathrm{GHz} \\ G_{3}^{+}\left(E_{4}\right)-G_{4}^{+}\left(E_{3}\right) & 1 \leftarrow 0 & (21,22) \leftarrow(1) & 17 & 759.9 \mathrm{GHz} \\ G_{4}^{-} / G_{3}^{+}\left(E_{2}\right) \leftarrow G_{3}^{-} / G_{4}^{+}\left(E_{1}\right) & 1 \leftarrow 1 & (23,24) \leftarrow(11,12) & 21 & 765.1 \mathrm{GHz} \\ G_{4}^{-} / G_{3}^{+}\left(E_{2}\right) \leftarrow G_{4}^{+}\left(E_{3}\right) & 1 \leftarrow 0 & (23,24) \leftarrow(4) & 19 & 1051.8 \mathrm{GHz} \\ G_{4}^{-} / G_{3}^{+}\left(E_{2}\right) \leftarrow G_{3}^{-}\left(E_{1}\right) & 1 \leftarrow 0 & (23,24) \leftarrow(3) & 20 & 1052.9 \mathrm{GHz} \\ G_{4}^{-} / G_{3}^{+}\left(E_{2}\right) \leftarrow G_{4}^{+}\left(E_{1}\right) & 1 \leftarrow 0 & (23,24) \leftarrow(1) & 4 & 1054.2 \mathrm{GHz}\end{array}$

(b)

\begin{tabular}{lcccc}
$G_{2}^{+} / G_{2}^{-}-G_{2}^{+} / G_{2}^{-}$ & $1 \leftarrow 1$ & $(9,10) \leftarrow(3,4)$ & 45 & $486.8 \mathrm{GHz}^{\mathrm{a}}$ \\
$G_{2}^{+} \leftarrow G_{2}^{+} / G_{2}^{-}$ & $0 \leftarrow 1$ & $(11) \leftarrow(3,4)$ & 20 & $500.7 \mathrm{GHz}$ \\
$G_{2}^{-} \leftarrow G_{2}^{+} / G_{2}^{-}$ & $0 \leftarrow 1$ & $(12) \leftarrow(3,4)$ & 22 & $503.1 \mathrm{GHz}$ \\
$G_{2}^{+} / G_{2}^{-} \leftarrow G_{2}^{-}$ & $1 \leftarrow 0$ & $(9,10) \leftarrow(2)$ & 26 & $597.4 \mathrm{GHz}$ \\
$G_{2}^{+} / G_{2}^{-}-G_{2}^{+}$ & $1 \leftarrow 0$ & $(9,10) \leftarrow(1)$ & 31 & $600.7 \mathrm{GHz}$ \\
$G_{2}^{+} \leftarrow G_{2}^{-}$ & $0 \leftarrow 0$ & $(11) \leftarrow(2)$ & 9 & $611.3 \mathrm{GHz}^{\mathrm{c}}$ \\
$G_{2}^{-} \leftarrow G_{2}^{-}$ & $0 \leftarrow 0$ & $(11) \leftarrow(1)$ & 24 & $613.7 \mathrm{GHz}^{\mathrm{b}}$ \\
$G_{2}^{+}-G_{2}^{+}$ & $0 \leftarrow 0$ & $(12) \leftarrow(2)$ & 23 & $614.6 \mathrm{GHz}^{\mathrm{b}}$ \\
$G_{2}^{-} \leftarrow G_{2}^{+}$ & $0 \leftarrow 0$ & $(12)-(1)$ & 8 & $617.0 \mathrm{GHz}^{\mathrm{c}}$ \\
$G_{2}^{+} / G_{2}^{-} \leftarrow G_{2}^{+} / G_{2}^{-}$ & $2 \leftarrow 2$ & $(15,16) \leftarrow(7,8)$ & 16 & $638.2 \mathrm{GHz}$ \\
$G_{2}^{+} / G_{2}^{-} \leftarrow G_{2}^{-} / G_{2}^{+}$ & $1 \leftarrow 1$ & $(13,14) \leftarrow(5,6)$ & 60 & $747.2 \mathrm{GHz}$ \\
$G_{2}^{+} / G_{2}^{-} \leftarrow G_{2}^{-}$ & $1 \leftarrow 0$ & $(13,14) \leftarrow(2)$ & 28 & $975.9 \mathrm{GHz}$ \\
$G_{2}^{+} / G_{2}^{-} \leftarrow G_{2}^{+}$ & $1 \leftarrow 0$ & $(13,14) \leftarrow(1)$ & 31 & $979.2 \mathrm{GHz}$ \\
$G_{2}^{+} / G_{2}^{-} \leftarrow G_{2}^{+} / G_{2}^{-}$ & $2 \leftarrow 1$ & $(15,16)-(3,4)$ & 49 & $1041.6 \mathrm{GHz}$ \\
& & $(c)$ & & \\
$A_{2}^{+} \leftarrow B_{2}^{+}$ & $0 \leftarrow 0$ & $(4) \leftarrow(1)$ & 22 & $483.3 \mathrm{GHz}$ \\
$A_{2}^{+} \leftarrow B_{2}^{+}$ & $1 \leftarrow 1$ & $(5,6) \leftarrow(2,3)$ & 36 & $518.5 \mathrm{GHz}$ \\
$A_{2}^{+} \leftarrow B_{2}^{+}$ & $1 \leftarrow 0$ & $(5,6) \leftarrow(1)$ & 31 & $729.0 \mathrm{GHz}$ \\
\hline \hline
\end{tabular}

Transitions in this subband were observed independently by Zwart (Ref. 23).

'This subband was previously reported by Havenith et al. (Ref. 21 ).

${ }^{c} Q$ branch only (due to Coriolis mixing of $K_{a}=0$ upper states with $K_{a}=1$ states).

$c[J(J+1)-2]^{1 / 2}$. The $c$ constants so determined are labeled by the numbers of the manifolds they couple. The form of the Coriolis coupling matrix elements is chosen to be equivalent to that of the Coriolis term given by Brocks et $a l^{32}$ in their quantum prescription for modeling the intermolecular dynamics of van der Waals's dimers:

$$
\begin{gathered}
\delta_{\Omega^{\prime}, \Omega+1} B[j(j+1)-\Omega(\Omega+1)]^{1 / 2} \\
\times[J(J+1)-\Omega(\Omega+1)]^{1 / 2},
\end{gathered}
$$

where $B$ is the rotational constant of the complex [the same $B$ used in expression (1) and (2) above], $j$ is the angular momentum from rotation of the monomers, $J$ is the total angular momentum, and $\Omega$ is the projection of total angular momentum along the a inertial axis. In this formalism $\Omega$ is equivalently the projection of both $j$ and $J$ onto the a axis, and when this Coriolis analysis is appropriate, we treat our quantum number $K_{a}$ as $\Omega$. This matrix element couples pure free internal rotor basis functions ${ }^{32}$ of the 
TABLE III. Rotational constants and relative energies as defined in Eqs. (1) and (2) for the (a) $E+E$, (b) $A+E$, and (c) $A+A$ states.

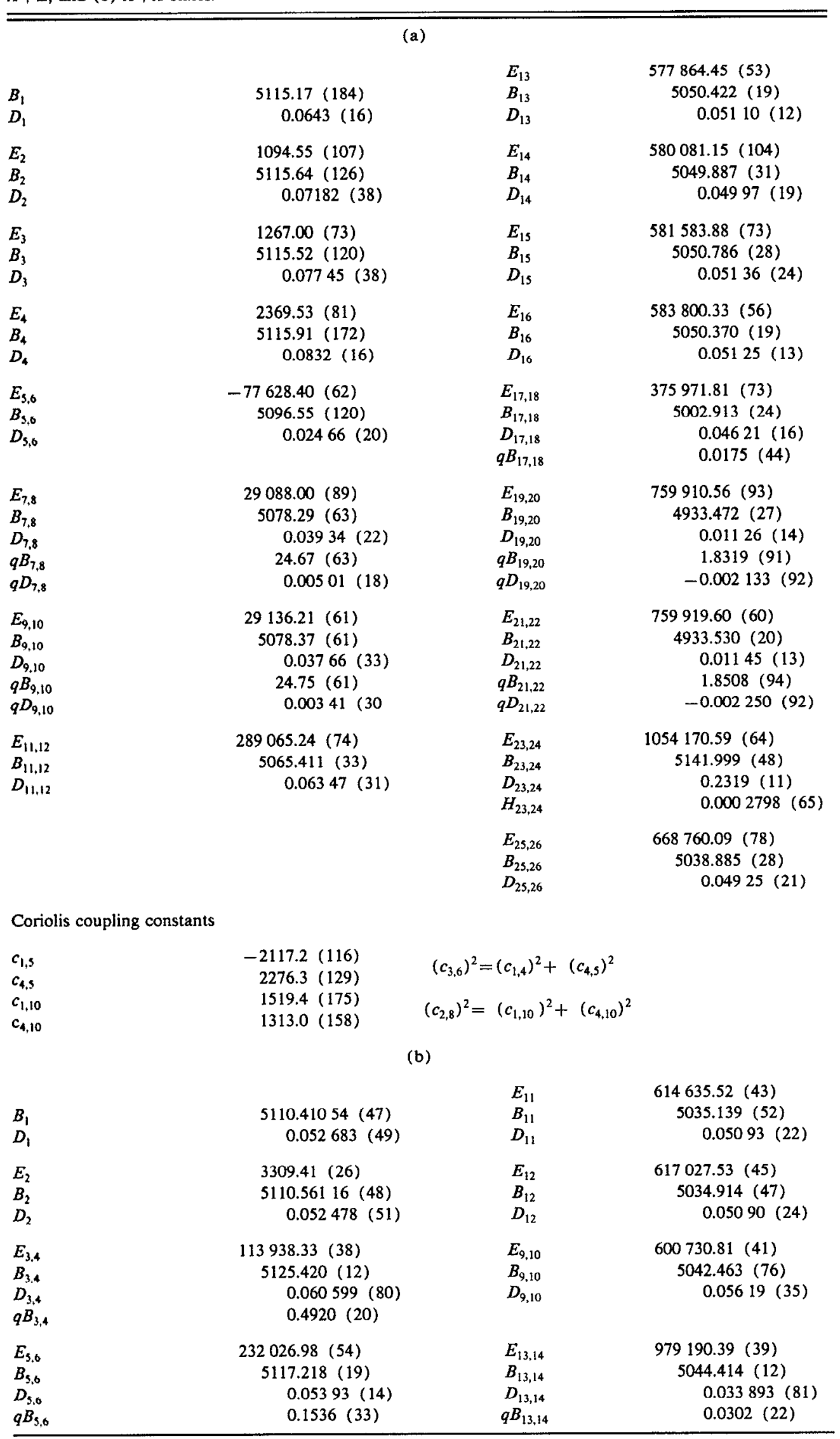


TABLE III. (Continued.)

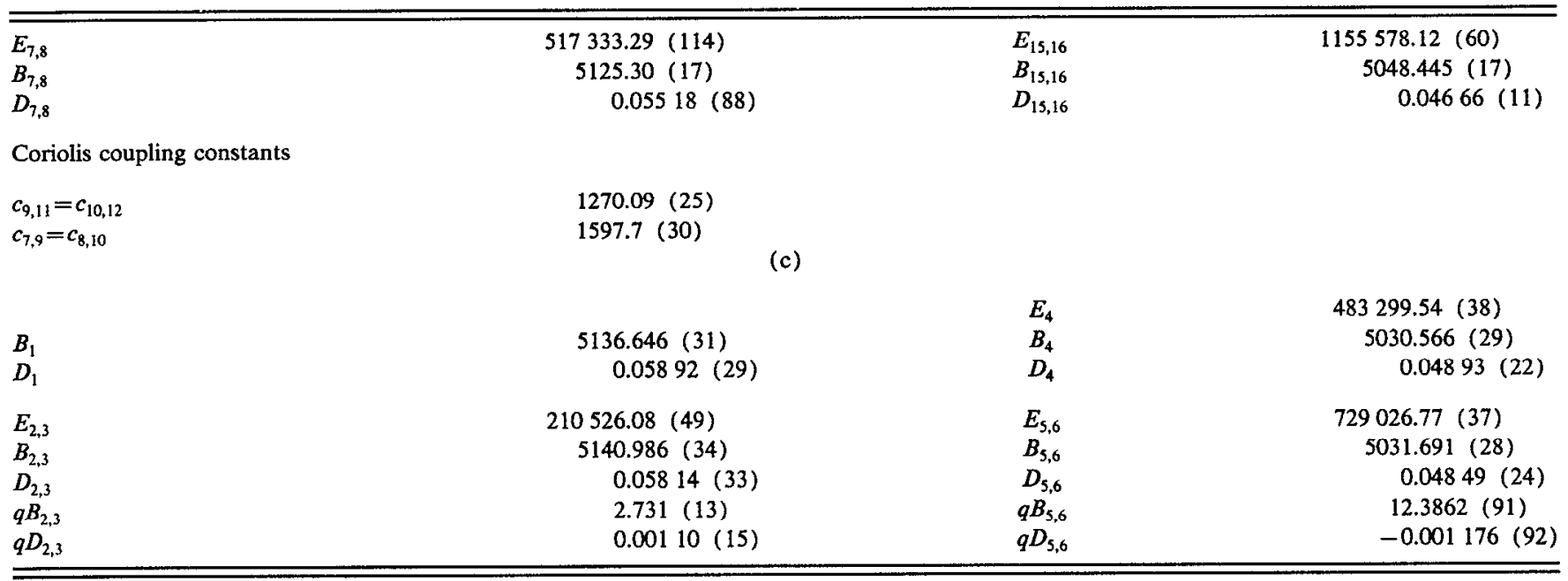

same internal rotor quantum numbers but different values of $\Omega$. The actual VRT states of the ammonia dimer are expected to be mixtures of many free internal rotor states, so that the Coriolis matrix element between any two ammonia dimer states should be smaller than the free internal rotor limit given above. This is consistent with the magnitudes of the coupling coefficients determined in our fits.

We determined one of the Coriolis coupling coefficients in the lowest $E+E$ states to be negative. This nonphysical result is an unresolved point of inconsistency in the analysis. This problem involves four of the $E+E$ VT states (states $1,4,5$, and 10 ) that are observed to have the same symmetry in the lowest $E+E$ states. To clarify this discussion, we present a "close-up" diagram of only the lowest $J=1 E+E$ states in Fig. 4. (The symmetry labels indicated in Fig. 4 will be discussed later in this paper.) Dashed lines show all possible $K_{a}=0-K_{a}=1$ Coriolis interactions between the states $1,4,5$, and 10 . We determined all of the corresponding coupling constants in our fit. It is easy to show that all four of these states involved do mutually interact, as indicated by the dashed lines in Fig. 4, by comparing them to the pair of states 2 and 8 and the pair of states 3 and 6 . States 8 and 10 are both upper components of $K_{a}=1 \mathrm{~K}$-type doublets, and their rotational energy-level spacings are nearly indistinguishable at our cxpcrimental accuracy. State 8 is strongly perturbed through a Coriolis interaction with state 2 ; however, neither state 1 nor state 4 couples to state 10 in the same way. Similarly, states 6 and 5 are the upper and lower components of a $K_{a}=1 \mathrm{~K}$-type doublet that is just barely split at high $J$. State 6 is strongly coupled to state 3 , yet neither state 1 nor state 4 couples to state 5 in the same way. Further, we note that the deviation of the rotational spacings in state 1 from the rotational spacings in state 2 is equal and opposite to the deviation of the rotational spacings in state 4 from the rotational spacings in state 3 . (This can be verified from Table III using simple arithmetic.) We know that this effect is not due to the direct coupling of state 1 to state 4 because they shift towards each other rather than further apart, and because states 13 and 16 , two higher $K_{a}=0$ states that are also very close in energy and have the same symmetry, do not interact. Therefore, we suggest that states 1 and 4 are coupled indirectly via interactions with the nearby $K_{a}=1$ states 5 and 10 . In our analysis, we assume that Coriolis interactions are responsible for this coupling.

After we remove the effects of the strongest Coriolis interactions betwecn $K_{a}=0$ and $K_{a}=1$, we find that the fitted $B_{n}$ constants consistently cluster around different values for the three groups of tunneling states, and also that the $B_{n}$ values of the "upper" states are consistently about $1 \%$ smaller than the "lower" state $B_{n}$ values. Without this explicit Coriolis treatment, variations in the observed values of $B_{n}$ would appear to be random. The rotational constants we determined after including the Coriolis interactions between $K_{a}=0$ and $K_{a}=1$ are still not pure "deperturbed" constants. Our data show evidence of strong Coriolis interactions between $K_{a}=1$ and $K_{a}=2$ as well, and we have not yet assigned all the $K_{a}=2$ states involved.

\section{B. Molecular symmetry group and symmetry assignment}

The appropriate choice for the molecular symmetry group of the ammonia dimer depends on which hydrogen tunneling motions occur on the time scale of our experiment. The most complete possible molecular symmetry group for the ammonia dimer is the permutation-inversion group $G_{144}{ }^{33}$ which includes all operations that permute the three protons on each ammonia subunit, operations that permute the two ammonia subunits, and spatial inversion. Only permutations that correspond to breaking chemical $\mathrm{N}-\mathrm{H}$ bonds are excluded from $G_{144}$. We assume that the ammonia dimer equilibrium structure contains a plane of symmetry, because there is no evidence that contradicts this simplifying assumption. Three kinds of tunneling motions can then be represented by the operations in $G_{144}$ : interchange of the two inequivalent ammonia 
TABLE IV. Relative energies(MHz) of observed (a) $E+E$, (b) $A+E$, and (c) $A+A$ states up to $J=5$.

\begin{tabular}{llllllllll}
\hline \hline$(n)$ & Sym. & $J$ & $K a$ & Rel. energy & $(n)$ & Sym. & $J$ & $K a$ & Rel. energy
\end{tabular}

(a)

$\begin{array}{ll}\text { (1) } & G 4+(E 3) \\ (1) & G 3-(E 4) \\ (1) & G 4+(E 3) \\ (1) & G 3-(E 4) \\ (1) & G 4+(E 3) \\ (1) & G 3-(E 4) \\ \text { (2) } & G 4-(E 3) \\ (2) & G 3+(E 4) \\ (2) & G 4-(E 3) \\ (2) & G 3+(E 4) \\ (2) & G 4-(E 3) \\ (2) & G 3+(E 4) \\ (3) & G 3-(E 1) \\ (3) & G 4+(E 1) \\ (3) & G 3-(E 1) \\ (3) & G 4+(E 1) \\ (3) & G 3-(E 1) \\ (3) & G 4+(E 1) \\ (4) & G 4+(E 1) \\ (4) & G 3-(E 1) \\ (4) & G 4+(E 1) \\ (4) & G 3-(E 1) \\ (4) & G 4+(E 1) \\ (4) & G 3-(E 1) \\ & \end{array}$

$\begin{array}{ll}0 \\ 1 \\ 2 \\ 3 \\ 4 \\ 5 \\ 0 \\ 1 \\ 2 & \\ 3 & \\ 4 & \\ 5 & \\ 0 & \\ 1 & \\ 2 & \\ 3 & \\ 4 & 0 \\ 5 & \\ 0 & \\ 1 & 0 \\ 2 & 0 \\ 3 & 0 \\ 4 & 0 \\ 5 & 0\end{array}$

0.0
10159.7
30345.0
60442.9
100466.2
150473.1

$\begin{array}{ll}(13) & G 3+(E 4) \\ (13) & G 4-(E 3) \\ (13) & G 3+(E 4) \\ (13) & G 4-(E 3) \\ (13) & G 3+(E 4) \\ (13) & G 4-(E 3)\end{array}$

0
1
2
3
4
5

577864.4

1094.5
11040.1
30944.5
60830.9
100726.3
150657.3

(14)

$G 3-(E 4)$

(14)

$G 4+(E 3)$

(14)

$G 3-(E 4)$

(14)

$G 4+(E 3)$

(14) $G 3-(E 4)$

(14) $G 4+(E 3)$

587965.1

608165.1

638462.2

678852.4

(15) $G 4-(E 2)$

1267.0
11741.8

32684.6

64081.7

(15)

(15)

$G 3+(E 2)$

105914.4

158159.6

(15)

$G 4-(E 2)$

$G 3+(E 2)$

(15)

$G 4-(E 2)$

2369.5

$G 3+(E 2)$

729331.1

12630.9

33294.2

64481.8

106189.3

158361.9

(16)

$G 3+(E 2)$

(16)

$G 4-(E 2)$

(16)

$G 3+(E 2)$

(16)

$G 4-(E 2)$

(16)

$G 3+(E 2)$

(16)

$G 4-(E 2)$

$-67679.5$

(17)

$G 3+(E 2)$

(5)

$G 3-, G 4+(E 1)$

$-47777.3$

(18)

$G 4-(E 2)$

$-47777.2$

$G 3-(E 1)$

(5)

$G 3-(E 1)$

(6)

$G 4+(E 1)$

(5)

(6)

$G 4+(E 1)$

$G 3-(E 1)$

$G 3-(E 1)$

(6)

$G 4+(E 1)$

$-17913.1$

$-17912.9$

21924.5

21924.9

71749.0

71749.5

(17)

(18)

$G 4-(E 2)$

(17)

(18)

$G 3+(E 2)$

$G 3+(E 2)$

(18)

$G 4-(E 2)$

$G 4-(E 2)$

(17)

$G 3+(E 2)$

(18)

$G 3+(E 2)$

39195.0

$G 4-(E 3)$

(8)

$G 3+(E 4)$

$G 3+(E 4)$

39579.2

59408.1

60545.8

89724.9

91959.5

130142.5

133785.7

180656.4

185987.6

(19)

(20)

G4-(E2)

580081.2

590180.7

610378.7

640672.6

681058.9

731532.8

581583.9

591685.2

611886.7

642185.9

682579.1

733061.2

583800.3

593900.9

614100.7

644397.4

684787.2

735265.3

385977.4

385977.5

405987.5

405987.7

435999.9

436000.3

476011.2

476011.9

526017.1

526018.1

769773.8

769781.1

789500.1

789521.9

819088.9

819132.3

858539.7

858611.3

907851.6

907957.6

769782.9

769790.3

785509.3

789531.4

819098.4

819142.2

858549.5

858621.7

907861.7

133830.0

180707.8

186030.4

(22)

(21)

$G 3-(E 4)$

$G 4+(E 3)$

(22)

G3-(E4)

907968.7

$(11,12) \quad G 4+, G 3-(E 1)$

$(11,12) \quad G 3-, G 4+(E 1)$

$(11,12) \quad G 4+, G 3-(E 1)$

$(11,12) \quad G 3-, G 4+(E 1)$

$(11,12) \quad G 4+, G 3-(E 1)$

299195.8

319455.4

$(23,24)$

$G 4-, G 3+(E 2)$

349841.0

$(23,24)$

$G 3+, G 4-,(E 2)$

1064453.7

390348.1

$(23,24) \quad G 4-, G 3+(E 2)$

$(23,24)$

$G 3+, G 4-(E 2)$

440970.5

$(23,24)$

$G 4-, G 3+(E 2)$

1085014.3

1115841.7

1156920.1

1208229.5

$(25,26) \quad G 3+, G 4-$

698991.6 
TABLE IV. (Continued.)

\begin{tabular}{|c|c|c|c|c|c|c|c|c|c|}
\hline$(n)$ & Sym. & $J$ & $K a$ & Rel. energy & $(n)$ & Sym. & $J$ & $K a$ & Rel. energy \\
\hline & & & & & $(25,26)$ & $G 3+, G 4-$ & 4 & 2 & 769518.1 \\
\hline
\end{tabular}

(b)

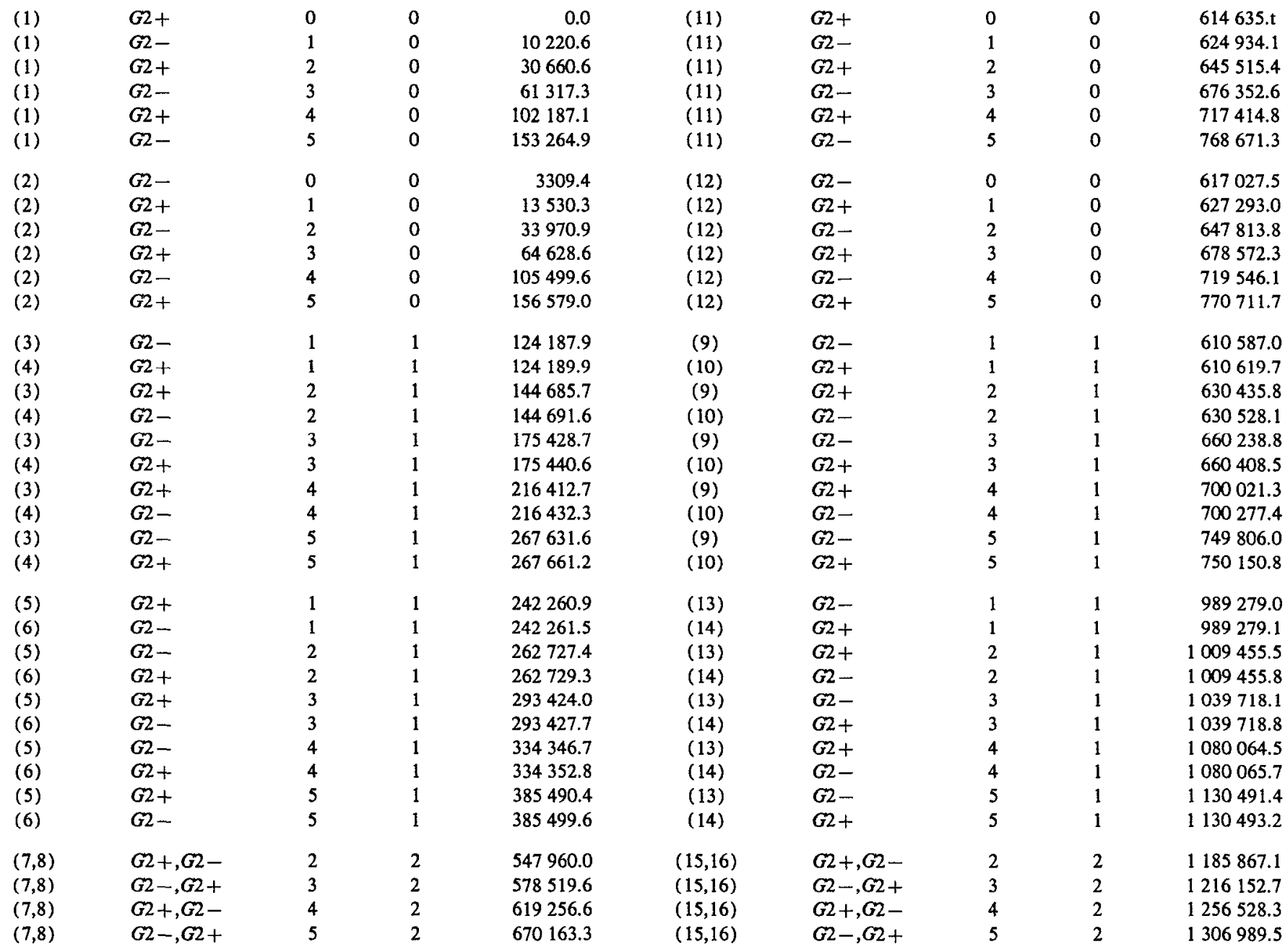

\begin{tabular}{|c|c|c|c|c|c|c|c|c|c|}
\hline (1) & $A 2+$ & 0 & 0 & 0.0 & (4) & $B 2+$ & 0 & 0 & 483299.5 \\
\hline (1) & $B 2-$ & 3 & 0 & 61631.3 & (4) & $A 2-$ & 3 & 0 & 543659.3 \\
\hline (1) & $A 2+$ & 4 & 0 & 102709.4 & (4) & $B 2+$ & 4 & 0 & 583891.3 \\
\hline (2) & $A 2+$ & 1 & 1 & 220802.4 & (5) & $B 2+$ & 1 & 1 & 739065.2 \\
\hline (3) & $B 2-$ & 1 & 1 & 220813.3 & (6) & $A 2-$ & 1 & 1 & 739114.7 \\
\hline (2) & $A 2+$ & 3 & 1 & 272176.6 & (5) & $B 2+$ & 3 & 1 & 789251.6 \\
\hline (3) & $B 2-$ & 3 & 1 & 272242.5 & (6) & $A 2-$ & 3 & 1 & 789548.5 \\
\hline (2) & $B 2-$ & 4 & 1 & 313267.5 & (5) & $A 2-$ & 4 & 1 & 829393.9 \\
\hline (3) & $A 2+$ & 4 & 1 & 313377.6 & (6) & $B 2+$ & 4 & 1 & 829888.4 \\
\hline (2) & $A 2+$ & 5 & 1 & 364620.4 & (5) & $B 2+$ & 5 & 1 & 879563.3 \\
\hline (3) & $B 2-$ & 5 & 1 & 364786.2 & (6) & $A 2-$ & 5 & 1 & 880304.4 \\
\hline
\end{tabular}




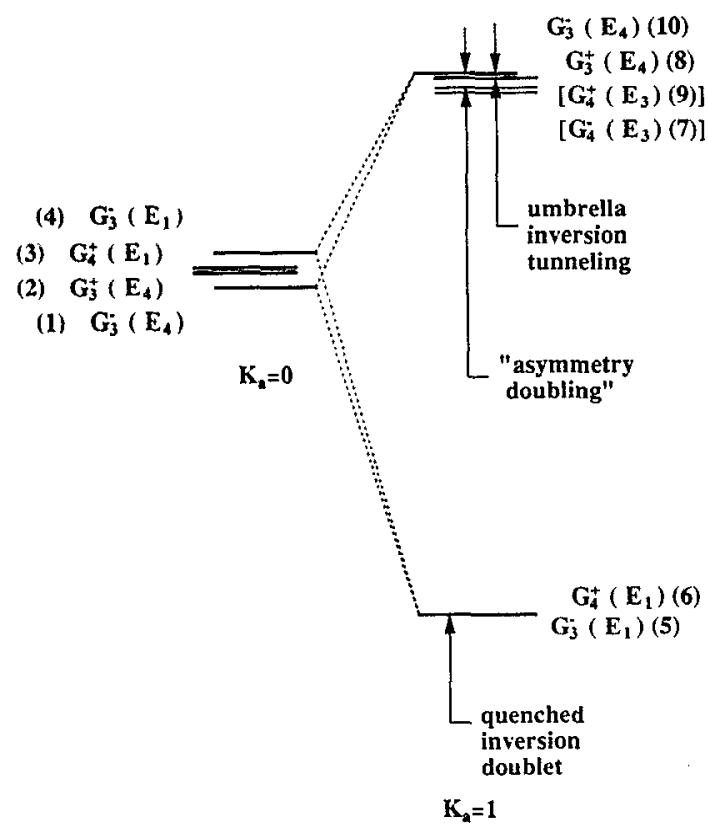

FIG. 4. Enlarged energy-level diagram for the lowest $J=1 E+E$ states. The observed Coriolis interactions within the $G_{3}^{-}$states (1), (4), (5), and (10) are indicated with dashed lines. We also observed Coriolis coupling between the $G_{4}^{+}$states (3) and (6) as well as between the $G_{3}^{+}$states (2) and (8). The $K_{a}=1 G_{4}^{-}$state (7) and $G_{4}^{+}$state (9) are unperturbed by $K_{a}=0$ states. The Coriolis perturbations are discussed in the rotational analysis section of the text and the symmetry assignments are discussed in the $E+E$ symmetry analysis section.

monomers (the donor and the acceptor), internal rotation of the monomers about their $C_{3}$ symmetry axes, and umbrella inversion tunneling of each monomer. A subgroup of $G_{144}$, the permutation inversion group $G_{36}$, was used in the analysis of Nelson and Klemperer. ${ }^{10}$ This group does not account for the umbrella inversion tunneling, and was used because it was assumed that the umbrella inversion tunneling would be totally quenched by the anisotropy in the ammonia dimer potential-energy surface. Perhaps surprisingly, we deduce from our data that $G_{144}$ is, in fact, the appropriate molecular symmetry group for the ammonia dimer. Nevertheless, it is useful to examine the data in terms of both $G_{36}$ and $G_{144}$ descriptions.

In order to interpret the ammonia dimer VRT spectrum, we must determine how many tunneling states arise from each vibration-rotation state of the dimer, and we must label them with irreducible representations of the molecular symmetry group. This will allow us to predict the number of VRT states that will be spectroscopically "connected" for each vibration-rotation state of the ammonia dimer. The molecular symmetry group of the rigid, nontunneling complex is $C_{s}$. The correlation between $C_{s}$ and $G_{36}$ has been derived previously by Nelson and Klemperer. ${ }^{10}$ They showed that, in $G_{36}$, there are eight tunneling sublevels associated with each rotational level of the rigid $C_{s}$ complex,

$$
\begin{aligned}
A^{\prime}= & A_{1}(66) \oplus A_{4}(78) \oplus G(144) \oplus G(144) \oplus E_{1}(36) \\
& \oplus E_{2}(36) \oplus E_{3}(30) \oplus E_{4}(42), \\
A^{\prime \prime}= & A_{2}(78) \oplus A_{3}(66) \oplus G(144) \oplus G(144) \oplus E_{1}(36) \\
& \oplus E_{2}(36) \oplus E_{4}(42) \oplus E_{3}(30) .
\end{aligned}
$$

The nuclear-spin statistical weights for the VRT levels of $\left({ }^{14} \mathrm{NH}_{3}\right)_{2}$ are given in parentheses after each irreducible representation. Also, Eqs. (3) are aligned so that the symmetry species of the even- and odd- $J$ end-over-end rotational levels of each of the eight tunneling sublevels are shown in the vertical columns. The selection rules for electric-dipole-allowed transitions, assuming molecular symmetry $G_{36}$, were found to be $A_{1} \leftrightarrow A_{3}, A_{2} \leftrightarrow A_{4}, G \leftrightarrow G$, $E_{1} \leftrightarrow E_{2}, E_{3} \leftrightarrow E_{3}$, and $E_{4} \leftrightarrow E_{4}$. Inspection of Eqs. (3) with these selection rules in mind reveals that transitions between either the $A$ or $E$ symmetry levels must involve a change in tunneling state while transitions between the $G$ symmetry levels may or may not involve a change in tunneling state. That is, for example, since $A_{1}$ and $A_{3}$ appear in different vertical columns in Eqs. (3), the frequency of a transition between a $J=0 A_{1}$ rotation-tunneling state and a $J=1 A_{3}$ rotation-tunneling state will include a contribution from tunneling as well as the usual rotational spacing. Even though rotation-tunneling states of $A_{1}$ and $A_{2}$ symmetry are not connected by electric dipole transitions, the energy separations between these states are purely rotational and are thus determined in the rotational fits. In $G_{36}$, the electric dipole selection rules separate the observed VRT levels of the ammonia dimer into four groups: (1) the $A_{1}, A_{2}, A_{3}$, and $A_{4}$ levels $(A+A$ states, we explain this nomenclature later in this section), (2) the $G$ levels $(A+E$ states), (3) the $E_{1}$ and $E_{2}$ levels ( $E+E$ states), and (4) the $E_{3}$ and $E_{4}$ levels (also $E+E$ states). All of the tunneling sublevels within each group are connected by electricdipole-allowed transitions, but transitions between tunneling sublevels in different groups are forbidden. We emphasize that, in $G_{36}$, there is only one tunneling sublevel of each $E$ symmetry type. Also, we note that there are two groups of $E+E$ states in $G_{36}$ and electric dipole transitions are not allowed between these two groups.

When umbrella inversion tunneling is considered feasible in the ammonia dimer, $G_{144}$ becomes the appropriate molecular symmetry group. Now, there are 22 tunneling sublevels associated with each rotational level of the rigid complex having $C_{s}$ symmetry, but only 14 tunneling sublevels have nonzero nuclear-spin statistical weight for $\left({ }^{14} \mathrm{NH}_{3}\right)_{2}$. We expect that the umbrella inversion tunneling motion has the highest barrier of all the tunneling motions we are considering for the ammonia dimer. Thus, we imagine that the more widely spaced $G_{36}$ tunneling states are further split by small umbrella inversion tunneling frequencies into the $G_{144}$ tunneling states. The correlation from $G_{36}$ to $G_{144}$ is 


$$
\begin{aligned}
& A_{1}=A_{1}^{+}(0) \oplus E^{-}(0) \oplus B_{2}^{+}(66), \\
& A_{2}=B_{1}^{-}(0) \oplus E^{+}(0) \oplus A_{2}^{-}(78), \\
& A_{3}=A_{1}^{-}(0) \oplus E^{+}(0) \oplus B_{2}^{-}(66), \\
& A_{4}=B_{1}^{+}(0) \oplus E^{-}(0) \oplus A_{2}^{+}(78), \\
& G=G_{1}^{+}(0) \oplus G_{1}^{-}(0) \oplus G_{2}^{+}(72) \oplus G_{2}^{-}(72), \\
& E_{1}=G_{3}^{-}(21) \oplus G_{4}^{+}(15), \\
& E_{2}=G_{3}^{+}(21) \oplus G_{4}^{-}(15), \\
& E_{3}=G_{4}^{+}(15) \oplus G_{4}^{-}(15), \\
& E_{4}=G_{3}^{+}(21) \oplus G_{3}^{-}(21) .
\end{aligned}
$$

Again, the nuclear-spin statistical weights for VRT levels of $\left({ }^{14} \mathrm{NH}_{3}\right)_{2}$ are shown in parentheses after the $G_{144}$ irreducible representations. So, the complete correlation between each $C_{s}$ rotational level and the $G_{144}$ tunneling sublevels is

$$
\begin{aligned}
A^{\prime}= & A_{1}^{+} \oplus B_{1}^{+} \oplus 2 E^{-} \oplus A_{2}^{+} \oplus B_{2}^{+} \oplus 2 G_{1}^{+} \oplus 2 G_{1}^{-} \oplus 2 G_{2}^{+} \\
& \oplus 2 G_{2}^{-} \oplus 2 G_{3}^{+} \oplus 2 G_{3}^{-} \oplus 2 G_{4}^{+} \oplus 2 G_{4}^{-} \\
A^{\prime \prime}= & B_{1}^{-} \oplus A_{1}^{-} \oplus 2 E^{+} \oplus B_{2}^{-} \oplus A_{2}^{-} \oplus 2 G_{1}^{-} \oplus 2 G_{1}^{+} \oplus 2 G_{2}^{-} \\
& \oplus 2 G_{2}^{+} \oplus 2 G_{4}^{-} \oplus 2 G_{4}^{+} \oplus 2 G_{3}^{-} \oplus 2 G_{3}^{+}
\end{aligned}
$$

Equations (5) are also aligned so that the vertical columns show the alternation of symmetry label with even and odd $J$. The electric dipole selection rules in $G_{144}$ are $A_{1}^{+} \leftrightarrow A_{1}^{-}$, $B_{1}^{+} \leftrightarrow B_{1}^{-}, \quad E^{+} \leftrightarrow E^{-}, \quad A_{2}^{+} \leftrightarrow A_{2}^{-}, \quad B_{2}^{+} \leftrightarrow B_{2}^{-}, \quad G_{1}^{+} \leftrightarrow G_{1}^{-}$, $G_{2}^{+} \leftrightarrow G_{2}^{-}, G_{3}^{+} \leftrightarrow G_{3}^{-}$, and $G_{4}^{+} \leftrightarrow G_{4}^{-}$. Pure rotational transitions are allowed in the $G_{1}^{+}, G_{1}^{-}, G_{2}^{+}, G_{2}^{-}, E^{+}$, and $E^{-}$ levels, but transitions between all other rotation-tunneling levels require a change in tunneling state. In $G_{144}$, the $A+A$ states are the $A_{1}^{+}, A_{1}^{-}, B_{1}^{+}, B_{1}^{-}, E^{+}, E^{-}, A_{2}^{+}, A_{2}^{-}, B_{2}^{+}$, and $B_{2}^{-}$levels, the $A+E$ states are the $G_{1}^{+}, G_{1}^{-}, G_{2}^{+}$, and $G_{2}^{-}$ levels, and the $E+E$ states are the $G_{3}^{+}, G_{3}^{-}, G_{4}^{+}$, and $G_{4}$ levels. Since some of these have zero statistical weight for $\left({ }^{14} \mathrm{NH}_{3}\right)_{2}$, we expect to observe only three groups of ammonia dimer VRT levels in $G_{144}$ : (1) the $A_{2}^{+}, A_{2}^{-}, B_{2}^{+}$, and $B_{2}^{-}$levels ( $A+A$ states), (2) the $G_{2}^{+}$and $G_{2}^{-}$levels $(A+E$ states), and (3) the $G_{3}^{+}, G_{3}^{-}, G_{4}^{+}$, and $G_{4}^{-}$levels $(E+E$ states). We emphasize that, in $G_{144}$ but not in $G_{36}$, there are two tunneling sublevels of each $E+E$ symmetry type, and all the $E+E$ tunneling manifolds can be connected together by electric-dipole-allowed transitions. Later, we exploit these important differences between the grouptheoretical description of the $E+E$ states in $G_{36}$ and that of the $E+E$ states in $G_{144}$ to assign the appropriate molecular symmetry group for the ammonia dimer.

It is useful to explore the correlation between the tunneling sublevels of the ammonia dimer and the rotational states of two ammonia monomers. The ammonia monomer equilibrium geometry has a threefold axis of symmetry, so the rotational states are classified as $A$ or $E$, for $k_{c}(\bmod 3)$ $=0$ and $k_{c}(\bmod 3) \neq 0$, respectively. There are also two proton spin states, $I=3 / 2$ (ortho) and $I=1 / 2$ (para) of $A$ and $E$ symmetry, respectively, which pair with the $A$ and $E$ rotational states. This leads to the well-known fact that electric dipole transitions between the states of $A$ and $E$ symmetry are forbidden. This selection rule also applies to the dimer complex when internal rotation tunneling is feasible: it leads to the three separate groups of $G_{144}$ tunneling states that we discussed above. In the accompanying paper, van Bladel et $a l^{22}$ refer to the $A+A$ states as ortho-ortho states, the $A+E$ states as ortho-para states, and the $E+E$ states as para-para states. If there is a very low barrier to internal rotation in the ammonia dimer, as in the more strongly hydrogen-bonded $\mathrm{HOH}-\mathrm{NH}_{3}$ dimer where the barrier to internal rotation of the ammonia acceptor was determined by Stockman et al. ${ }^{34}$ to be $\$ 10 \mathrm{~cm}^{-1}$, a correlation between dimer internal rotation states and monomer rotational states is directly useful for estimating the relative internal rotation splittings in the ammonia dimer. Since the ammonia monomers in the ammonia dimer can interchange their donor and acceptor roles, the average barrier to internal rotation in the ammonia dimer is expected to be higher than in $\mathrm{HOH}-\mathrm{NH}_{3}$. If the ammonia rotors are substantially hindered so that a high barrier analysis of the tunneling splittings is appropriate - such a formalism has recently been described by Coudert and Hougen $^{35}$ - the correlation between dimer tunneling states and monomer rotational states is still useful for establishing upper limits for the relative internal rotation tunneling splittings in the ammonia dimer.

In the calculations described in the accompanying paper, van Bladel et $a l^{22}$ have used basis functions formed from linear combinations of coupled monomer symmetric rotor wave functions. ${ }^{31}$ These basis functions have been symmetrized so that they transform according to irreducible representations of $G_{36}$. The true VRT states of the ammonia dimer are not described by the pure (or "free") symmetrized basis functions; rather, they are mixed by anisotropy (long-range electrostatic, induction and dispersion interactions, short-range exchange forces, etc.) in the intermolecular potential-energy surface. The description of the true mixed VRT states of the ammonia dimer is the ultimate goal in the calculations of van Bladel et al. Nevertheless, the free internal rotor limit has been used by Nelson and Klemperer ${ }^{10}$ to illustrate the energetics and internal rotation dynamics due to the internal rotation of the ammonia monomers about their $C_{3}$ axes. We describe the free-rotor components of some of the lowest-energy $G_{36}$ basis functions and the $G_{144}$ analogs for total $J=0$ below.

The $A_{1}$ and $A_{4}$ tunneling states of $G_{36}$, or the $A_{1}^{+}, B_{2}^{+}$, $B_{1}^{+}, A_{2}^{+}$, and $E^{-}$tunneling states of $G_{144}$, are constructed from ammonia monomer $A$ states. For example, the combination of two ammonia monomers in $0_{0}$ rotational states transforms like $A_{1}$ in $G_{36}$, and the combination of one monomer in $\mathrm{O}_{0}$ with the other in $1_{0}$ transforms as $A_{1} \oplus A_{4}$. In these lowest $A+A$ basis functions, there is no projection of internal angular momentum from internal rotation about the monomer $C_{3}$ symmetry axes. (Of course, the true VRT wave functions probably also contain contributions from $3_{3}$ states, coupled in by electrostatic interactions involving the ammonia monomer electric octopole moment 
as well as short-range repulsive contributions caused by steric effects from the three hydrogens on each ammonia monomer. However, the $3_{3}$ contributions will not be dominant in the lowest $A+A$ states.) To generalize from $G_{36}$ to $G_{144}$, we must include the umbrella inversion states of the monomers. There is a symmetric and antisymmetric inversion component for every monomer rotational state. In the $A$ states with $k_{c}=0$, only one component has nonzero statistical weight; in $0_{0}$, this is the antisymmetric inversion state $0_{0}^{-}$. The combination of two monomers in $0_{0}^{+}$transforms as $A_{1}^{+}$in $G_{144}$, which of course has zero statistical weight. One monomer in $0_{0}^{+}$plus one monomer in $0_{0}^{-}$gives $E^{-}$in $G_{144}$, which also has zero statistical weight. The combination of two monomers in $0_{0}^{-}$transforms as $B_{2}^{+}$in $G_{144}$, which we can observe spectroscopically.

The $G$ states of $G_{36}$, and the $G_{1}^{+}, G_{1}^{-}, G_{2}^{+}$, and $G_{2}^{-}$ states of $G_{144}$, are constructed from one monomer in an $A$ state plus one monomer in an $E$ state. The lowest-energy free monomer basis function of $G$ symmetry in $G_{36}$ involves one monomer in $0_{0}$ (an $A$ state) with the other in $1_{1}$ (an $E$ state). The fourfold degeneracy arises because this combination can be taken four ways: $\left(0_{0}+1_{+1}, 0_{0}+1_{-1}, 1_{+1}\right.$ $\left.+0_{0}, 1_{-1}+0_{0}\right)$. In the lowest $A+E$ states, the internal rotation angular momentum $(j=1$, in this example) is projected onto the $C_{3}$ axis of just one of the ammonia monomers. (More classically, we might say that the $E$ monomer is rotating about its symmetry axis, but the $A$ monomer is not rotating at all.) The relative energy of this lowest $A+E$ basis function with respect to the lowest $A+A$ basis function is equal to the rotational energy of the free $1_{1}$ ammonia monomer rotational state. In $G_{36}$, there are two $A+E$ tunneling states for each $C_{s}$ "vibrational" state of the complex. In the high barrier tunneling limit, it can be seen from the work of Coudert and Hougen, ${ }^{35}$ that the energy separation of these two $A+E$ tunneling states (in the absence of interchange tunneling) is equal to the difference in the hindered internal rotation frequencies of the inequivalent monomers. That is, the splitting between one $A+E$ state and the $A+A$ states is equal to the hindered internal rotation frequency of the donor and the splitting between the other $A+E$ state and the $A+A$ states is equal to the hindered internal rotation frequency of the acceptor. It is straightforward to generalize to $G_{144}$ combinations. Both the symmetric and antisymmetric inversion levels of an ammonia monomer $E$ rotational state have nonzero statistical weight. The combinations of one monomer in $0_{0}^{+}$ (which has zero statistical weight) with the other in $1_{1}^{+}$or $1_{1}^{-}$transform like $G_{1}^{+}$or $G_{1}^{-}$in $G_{144}$, and both of these symmetries of course have zero statistical weight. The combinations of one monomer in $0_{0}^{-}$with the other in $1_{1}^{+}$ or $1_{1}^{-}$transform like $G_{2}^{+}$or $G_{2}^{-}$in $G_{144}$, and both have nonzero statistical weight and thus are observable.

The $E_{1}, E_{2}, E_{3}$, and $E_{4}$ states of $G_{36}$, and the corresponding $G_{3}^{+}, G_{3}^{--}, G_{4}^{+}$, and $G_{4}^{-}$states of $G_{144}$, are constructed from combinations of two ammonia monomers in $E$ states. The transformation propertics of the corresponding basis functions are more complicated. For example, the lowest-energy $E+E$ combination, formed from two monomers in $1_{1}$ states, transforms like $2 E_{1} \oplus E_{2} \oplus 3 E_{3}$ in $G_{36}$.
The doubly degenerate combination of $\left(1_{+1}+1_{-1}, 1_{-1}\right.$ $\left.+1_{+1}\right)$ transforms as $E_{1}$ when the total internal angular momentum $(j)$ is 0 or 2, while it transforms as $E_{2}$ for $j=1$. The doubly degenerate combination of $\left(1_{+1}+1_{+1}, 1_{-1}\right.$ $+1_{-1}$ ) transforms like $E_{3}$ for all possible total internal angular momenta $j=0,1$, or 2 . In the $E+E$ states, the internal rotation angular momentum comes from the vector sum of the angular momentum from both monomers and can have a nonzero projection onto both monomer $C_{3}$ symmetry axes. Although we may expect the $E_{1}$ and $E_{2}$ tunneling sublevels to behave perhaps differently from the $E_{3}$ and $E_{4}$ sublevels, we see that they will still have roughly the same energy in the free internal rotor limit. The relative energy of the lowest $E+E$ basis functions with respect to the lowest $A+A$ function is equal to twice the rotational energy of the $1_{1}$ rotational energy of the free monomer. In the high barrier treatment of Hougen and Coudert, ${ }^{35}$ the splitting between the $G_{36} E+E$ states and the $A+A$ states is equal to the hindered internal rotation frequency of the donor plus the hindered internal rotation frequency of the acceptor. An additional splitting, which we expect to be small, between the $\left(E_{1}, E_{2}\right)$ and $\left(E_{3}, E_{4}\right)$ pairs can be related to the difference in frequencies of an internal rotation tunneling motion in which both monomers rotate together clockwise or counterclockwise about their $C_{3}$ symmetry axes and an internal rotation tunneling motion in which both monomers rotate together but in opposite senses.

Summarizing the above observations, we expect the internal rotor levels of the ammonia dimer to be separated into three equally spaced clumps: the lowest group would be the $A+A$ states, next, the $A+E$ states, and the highest group would be the $E+E$ states. If there were no barrier to internal rotation, the spacing between these groups would be equal to the $1_{1}$ rotational energy of the ammonia monomer. As the barriers to internal rotation become more appreciable, these spacings decrease to the hindered internal rotation frequencies of the complexed monomers, and the internal rotor tunneling states within the $A+E$ and $E+E$ groups start to split apart.

In their group-theoretical analysis, Nelson and Klemperer ${ }^{10}$ divided the correlation of rigid molecule rotational levels with the tunneling-rotational levels of the ammonia dimer into two steps, considering two ways to arrive at $G_{36}$ from $C_{s}$. One way, called the $2 C_{3}+I$ limit, assumes that the energy splittings caused by the internal rotation of the ammonia monomers about their $C_{3}$ symmetry axes are much larger that the splittings caused by the donoracceptor interchange tunneling. The pattern of ammonia dimer tunneling energy levels has previously been assumed to resemble this limit. ${ }^{9,21}$ In the $2 C_{3}+I$ limit, we would expect to find that the $A_{1}$ and $A_{4}(A+A), E_{1}$ and $E_{2}(E+E)$, and the $E_{3}$ and $E_{4}(E+E)$ pairs of tunneling sublevels are each split by small interchange tunneling frequencies. The pair of $G(A+E)$ sublevels can be split by a contribution from the internal rotation tunneling (the difference in internal rotation frequencies of the two inequivalent monomers) as well as the small interchange tunneling frequency. The pure rotational transitions discovered 


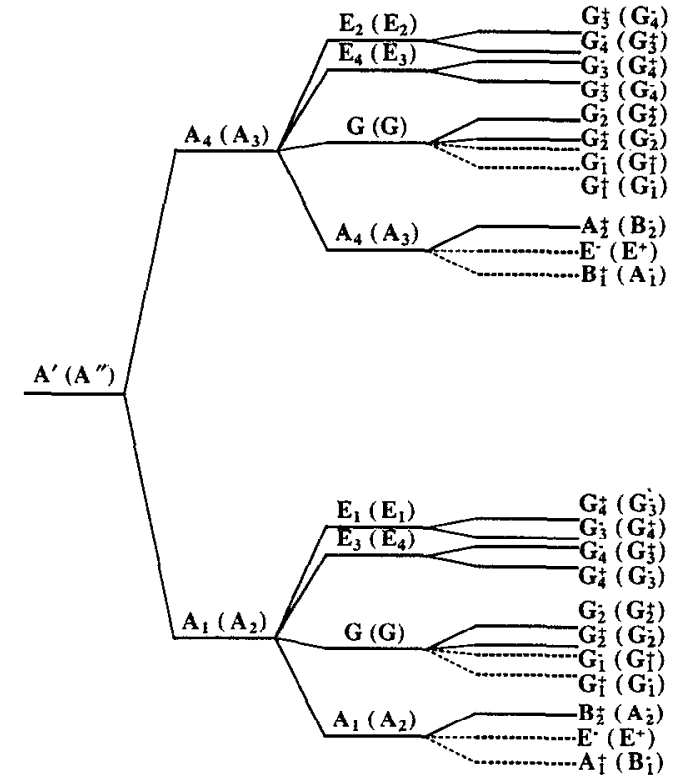

$\begin{array}{llll}\text { (a): } C_{5} & \text { (b): } G_{4} & \text { (c): } G_{36} & \text { (d): } G_{1+4}\end{array}$

FIG. 5. Correlation diagram for the tunneling levels of the ammonia dimer. Proceeding from the left-hand column, (a) one rotational state of the rigid complex with a plane of symmetry is spit into rotation tunneling sublevels as (b) the "donor-acceptor" interchange, (c) $C_{3}$ internal rotation, and (d) monomer umbrella inversion tunneling motions become feasible. The symmetry species of each rotation tunneling state in the appropriate molecular symmetry group for each column is indicated for even $J$ (odd $J$ ). See the text for the complete explanation.

by the Klemperer group were assigned to these $G$ sublevels. Our microwave search was initially undertaken in order to measure the rotation-interchange tunneling subbands in the $A+A$ and $E+E$ tunneling sublevels.

The opposite way of arriving at $G_{36}$ from $C_{s}$, called the $I+2 C_{3}$ limit, ${ }^{10}$ assumes that the interchange tunneling is much faster that the internal rotation. This turns out to be a better description of the now vastly expanded experimental results, and we illustrate this qualitative scheme in Fig. 5. As in Nelson and Klemperer's analysis, ${ }^{10}$ we discuss each column in this diagram, proceeding from left to right. In column (a) we show one rotational level of a rigid ammonia dimer with an equilibrium structure that contains a plane of symmetry. The appropriate molecular symmetry group in this case is $C_{s}$. In column (b) the donoracceptor interchange tunneling splits this rotational state into a symmetric tunneling sublevel and an antisymmetric tunneling sublevel. This new symmetry operation doubles the size of the molecular symmetry group, so that it is now $G_{4}$. In column (c) the internal rotation of each monomer about its $C_{3}$ symmetry axis causes an additional ninefold splitting of each symmetric or antisymmetric interchange tunneling sublevels into four distinct tunneling sublevels: an $A+A$, an $A+E$, and two $E+E$ states. These internal rotation sublevels are sketched assuming the energy ordering as described above. The internal rotation symmetry operations increase the size of the molecular symmetry group by a factor of $3 \times 3=9$ times, to give $G_{36}$. Finally, in column (d), the umbrella inversion of both monomers be- comes feasible, and increases the size of the molecular symmetry group by a factor of $2 \times 2=4$ times to give $G_{144}$.

The tunneling levels that we have accessed spectroscopically are connected by electric dipole transitions into three groups. It is straightforward, using the relative nuclear-spin statistical weights observed in the closely spaced $K$-type doublets, as well as the presence of the previously observed pure rotational transitions in the $A+E$ states, to assign these groups to the $A+A$ states, the $A+E$ states, and the $E+E$ states. Additional confirmation of the $A+A$ and $E+E$ assignment is simply the fact that more $E+E$ states were more observed than could possibly be expected for the $A+A$ states alone.

\section{The $E+E$ states}

The VRT levels identified as the $E+E$ states are shown in Fig. 3(a). A total of 509 observed electric dipole transitions connect these levels. ${ }^{31}$ Table II(a) lists all of the observed subband origins for the transitions connecting these $E+E$ states and serves as a quick summary of this subset of the spectrum. The fitted rotational constants, relative $J=0$ energies, and Coriolis coupling constants are shown in Table III(a), and the calculated lowest VRT energy levels $(J=0-5)$ from this fit are listed in Table III(a). We discuss the most interesting features of this data set below.

The most important observation to make about the observed $E+E$ states is that two of the four lower $K_{a}=0$ $E+E$ states have the same symmetry and two of the four upper $K_{a}=0 E+E$ states have the same symmetry, as we mentioned in our description of the Coriolis analysis above. We learned this by discovering four sets of $\left|\Delta K_{a}\right|=1$ transitions between two $K_{a}=0$ VRT states and a single $K_{a}=1$ VRT state. (These are the $K_{a}=0 \leftarrow 1$ subbands near 550 $\mathrm{GHz}$, the $K_{a}=0 \leftarrow 1$ subbands near $660 \mathrm{GHz}$, the $K_{a}=1 \leftarrow 0$ subbands near $760 \mathrm{GHz}$, and the $K_{a}=1 \leftarrow 0$ subbands near $1050 \mathrm{GHz}$.) This leads to two important conclusions, as illustrated in the abbreviated correlation diagram shown in Fig. 6. First, $G_{144}$ is the appropriate molecular symmetry group for the ammonia dimer. The $E+E$ states of $G_{36}$ are $E_{1}, E_{2}, E_{3}$, and $E_{4}$, and there is only one tunneling sublevel of each symmetry for each vibrational level. However, in $G_{144}$, the $E+E$ states are comprised of two $G_{3}^{+}$, two $G_{3}^{-}$, two $G_{4}^{+}$, and two $G_{4}^{-}$sublevels, which is consistent with our observations. Second, the interchange tunneling splitting must be large.

Consider the possible correlations of four $G_{144} E+E$ states to two $G_{36} E+E$ states. If, as in the $\left(2 C_{3}+I\right)$ limit shown in Fig. 6(a), the two $G_{36} E+E$ states were interchange tunneling partners such as the pair $E_{1}$ and $E_{2}$ or the pair $E_{3}$ and $E_{4}$, then the resulting $G_{144}$ states would be $G_{3}^{+}$, $G_{3}^{-}, G_{4}^{+}$, and $G_{4}^{-}$; these all have different symmetries. On the other hand, as in the $I+2 C_{3}$ limit shown in Fig. 6(b), if the two $G_{36} E+E$ states were $E_{1}$ and $E_{3}$, which are both symmetric with respect to interchange tunneling, then the resulting $G_{144}$ states would be $G_{3}^{-}, G_{4}^{+}, G_{4}^{+}$, and $G_{4}^{-}$; two of these have the same symmetry $G_{4}^{+}$, which is consistent with our observation of two pairs of nearby $K_{a}=0$ states with the same symmetry. If the four lowest $K_{a}=0 E+E$ 

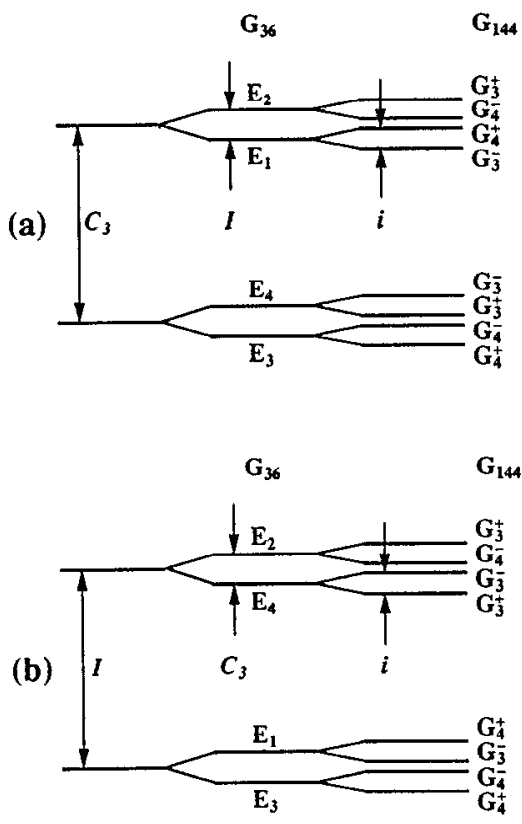

FIG. 6. (a) Correlation of $G_{36}$ (no monomer umbrella inversion) $E+E$ tunneling states in the $2 C_{3}+I$ limit to the $G_{144}$ (with monomer umbrella inversion) $E+E$ tunneling states. In this case, the $G_{144}$ states with the same symmetry are well separated. (b) Correlation of $G_{36} E+E$ tunneling states in the $I+2 C_{3}$ limit to the $G_{144} E+E$ tunneling states. In this case, two pairs of $G_{144}$ states with the same symmetry, the $G_{4}^{+}$states and the $G_{3}^{+}$states, lie close in energy. This tunneling scheme is consistent with our far-infrared spectra.

states are symmetric with respect to interchange tunneling, then four more $K_{a}=0 E+E$ states which are their antisymmetric interchange partners should exist. We did not find these interchange partners for the lower four $K_{a}=0$ $E+E$ states in our microwave search, nor have we assigned far-infrared subbands that might originate from the interchange partners of the lowest $E+E$ states; this suggests that the interchange tunneling splitting in the $E+E$ states is large. Later, we deduce that the antisymmetric $E+E$ interchange partners are actually the upper states of the $K_{a}=0 \leftarrow 0$ subbands we measured near $580 \mathrm{GHz}$. This suggests that the $I+2 C_{3}$ tunneling scheme described by Nelson and Klemperer ${ }^{10}$ is the most appropriate.

We place $G_{3}$ and $G_{4}$ labels on all the $E+E$ VRT states using nuclear-spin statistics, but we must make an educated choice between two possible overall assignments of + and - labels. The two lower $K_{a}=0 E+E$ states that have the same symmetry are either both $G_{4}^{+}$or both $G_{4}^{-}$. So the choices for the four lower $E+E K_{a}=0$ states are (1) $G_{4}^{+}$(from $E_{1}$ ), $G_{3}^{-}$(from $\left.E_{1}\right), G_{4}^{+}$(from $E_{3}$ ), and $G_{4}^{-}$ (from $\left.E_{3}\right)$; or (2) $G_{4}^{-}$(from $E_{2}$ ), $G_{3}^{+}$(from $E_{2}$ ), $G_{4}^{-}$ (from $E_{3}$ ), and $G_{4}^{+}$(from $E_{3}$ ). We prefer assignment (1) because all of these states are symmetric with respect to interchange tunneling. When interchange tunneling is large, as in the $I+2 C_{3}$ limit, the correlation diagram shows unambiguously that the $E_{1}$ and $E_{3} G_{36}$ states must be the lowest-energy $E+E$ states. In clusters such as $(\mathrm{HCl})_{2}$ and $(\mathrm{HF})_{2}$, in which interchange is the only possible tunneling motion, the symmetric interchange tunneling state is always lower in energy.
The pattern of $K_{a}=1 E+E$ tunneling states looks rather different from that in $K_{a}=0$. In addition, the differing $K_{a}=1$ behavior of these states further subdivides them into two groups. The $K_{a}=1$ VT manifolds labeled in Fig. 3 as $(5,6),(11,12),(17,18)$, and $(23,24)$ are spaced apart by 367,87 , and $678 \mathrm{GHz}$. But, as shown in Table II(a), the splitting between the two levels with the same $J$ within each of these four manifolds, which is reflected in our rotational constants $q B_{n}$, is essentially zero to within our experimental resolution, or very small. If there were no Coriolis interactions involving these states, the $q B_{n}$ values would be exactly zero. By contrast, the $K_{a}=1$ manifolds labeled in Fig. 3 as $(7,8),(9,10),(19,20)$, and $(21,22)$ are spaced apart by $48 \mathrm{MHz}, 731 \mathrm{GHz}$, and $9 \mathrm{MHz}$. The values of $q B_{n}$ for these levels, listed in Table II (b), are close to the valucs of the asymmetry parameter $\frac{1}{4}(B-C)$ that can be predicted from the proposed experimental structure (21 $\mathrm{MHz})$ or the proposed theoretical structure $(15 \mathrm{MHz})$. The effects of Coriolis interactions have already been removed from these values of $q B_{7,8}$ and $q B_{9,10}$ in Table III(b). The two qualitatively different observed Coriolis interactions of these $K_{a}=1 E+E$ states show how they correlate to the two different groups of $G_{36} E+E$ states.

First, let us consider the very-lowest-energy $K_{a}=1 E$ $+E$ tunneling manifold $(5,6)$. For this level, we found that $q B_{5,6}$ is zero. The $J=1 K_{a}=1$ states of this manifold have symmetry species $G_{3}^{-}(5)$ and $G_{4}^{+}(6)$, and they are shown in Fig. 4. The $G_{3}^{-} 1_{1}(5)$ state is pushed down in energy by a Coriolis interaction with the $G_{3}^{-} 1_{0}$ states ( 1 and 4) that are $\sim 78 \mathrm{GHz}$ higher in energy and the $G_{4}^{+} 1_{1}$ state (6) is pushed down in energy by a Coriolis interaction with the $G_{4}^{+} 1_{0}$ state (3) that is also $\sim 78 \mathrm{GHz}$ higher in energy. This is why the observed splitting of the $l_{1}$ states is so small. We might think of a pair of two $1_{1}$ rotational wave functions as $2^{-1 / 2}\left(\left|K_{a}=+1\right\rangle+\left|K_{a}=-1\right\rangle\right)$ and $2^{-1 / 2}\left(\left|K_{a}=+1\right\rangle-\left|K_{a}=-1\right\rangle\right)$, of which only the " + " linear combination can interact with $K_{a}=0$ states. But the observed Coriolis interactions show that this does not describe the $K_{a}=1$ states $(5,6)$. Rather, the + rotational states (or even simpler rotational states such as $\left.\left|K_{a}= \pm 1\right\rangle\right)$ of two different tunneling states are present in this $K_{a}=1$ pair. Since the two $1_{1}$ states have alternating intensities (due to different nuclear-spin statistical weights), their symmetries must be $G_{4}^{+}$and $G_{3}^{-}$, and the two states are the symmetric and antisymmetric umbrella inversion tunneling components of a $G_{36} E_{1}$ state. That is, the umbrella inversion tunneling splitting of the $G_{36} E_{1}$ sublevel is exactly zero in $K_{a}=1$. The $367 \mathrm{GHz}$ splitting of the $(5,6)$ and $(11,12)$ manifolds is not due to umbrella inversion tunneling; instead this large energy separation may be the result of coupling of a large internal angular momentum to the a inertial axis. This effect is also found in the $K_{a}=1 E$ states of $\mathrm{Ar}-\mathrm{NH}_{3}{ }^{29,36,37}$

Now, let us consider the example of the $K_{a}=1 E+E$ tunneling manifold $(9,10)$. We found that $q B_{9,10}$ is $\sim 25$ $\mathrm{MHz}$, and that the separation between the $(7,8)$ and $(9,10)$ manifolds is only $\sim 48 \mathrm{MHz}$. As shown in Fig. 4, the $J=1 K_{a}=1$ states in this manifold also have symmetry species $G_{4}^{+}(9)$ and $G_{3}^{-}(10)$. The $G_{3}^{-} 1_{1}$ state $(10)$ is 
pushed up in energy by a Coriolis interaction with the $G_{3}^{-}$ $1_{0}$ states ( 1 and 4$) \sim 30 \mathrm{GHz}$ lower in energy, but the $G_{4}^{+}$ $1_{1}$ state (9) does not interact with the $G_{4}^{+} 1_{0}$ state (3) also $\sim 30 \mathrm{GHz}$ lower in energy. This leads to the very large observed value of $q B_{9,10}$. The observed Coriolis interactions confirm that the $G_{3}^{-}$state $(10)$ can be thought of as a symmetric rotational state $\left[2^{-1 / 2}\left(\left|K_{a}=+1\right\rangle\right.\right.$ $\left.\left.+\left|K_{a}=-1\right\rangle\right)\right]$ because it can interact with the $K_{a}=0$ state and that the $G_{4}^{+}$state (9) can be thought of an antisymmetric rotational state $\left[2^{-1 / 2}\left(\left|K_{a}=+1\right\rangle-\left|K_{a}=-1\right\rangle\right)\right]$. For even $J$, the symmetric rotational states of manifold $(9,10)$ have symmetry $G_{4}^{+}$and the symmetric rotational states of manifold $(7,8)$ have symmetry $G_{4}^{-}$. Therefore the $K_{a}=1$ manifolds $(7,8)$ and $(9,10)$ are the symmetric and antisymmetric umbrella inversion tunneling components of a $G_{36} E_{3}$ tunneling state. And, the very small $\sim 48 \mathrm{MHz}$ energy difference between them is, in fact, the $K_{a}=1 \mathrm{um}$ brella inversion tunneling frequency.

We find that the electric dipole selection rules in $G_{36}$ can be viewed as very approximate additional selection rules for transitions between the $G_{144}$ VRT states. This means, for example, that we would expect to see $G_{3}^{+}\left(E_{2}\right) \leftarrow G_{3}^{-}\left(E_{1}\right)$ but not $G_{3}^{+}\left(E_{4}\right)-G_{3}^{-}\left(E_{1}\right)$. We also find, however, that the Coriolis coupling between $K_{a}=0$ and $K_{a}=1$ levels almost completely spoils the approximate $G_{36}$ selection rules. That is, we observe subbands that would be forbidden by the $G_{36}$ selection rules when either the lower or upper state is affected by strong Coriolis interactions. These extra observed subbands allow us to determine, for example, the energy difference between the $G_{36}$ $E_{1}$ level and the $G_{36} E_{3}$ level. The observed transitions that defy the $G_{36}$ selection rules are often weaker than similar transitions that obey the $G_{36}$ selection rules. We use these observations to assign the $G_{36}$ symmetries of the $K_{a}=0$ $E+E$ states, since we know the $G_{36}$ symmetries of the $K_{a}=1$ states. We indicate both the $G_{144}$ and $G_{36}$ symmetries in Fig. 3(a) and in Ref. 31 and Tables II(a) and IV (a).

The tunneling rotation subband that we discovered in the microwave search also involves the $E+E$ states. The observed transitions in this subband are indicated in Fig. 2(b). This $K_{a}=1 \leftarrow 1$ subband with a band origin at 48 MHz, between states $(7,8)$ and $(9,10)$, directly measures the umbrella inversion tunneling splitting of the $K_{a}=1 G_{36}$ $E_{3}$ level. Although these transitions obey the approximate $G_{36}$ selection rules as well as the rigorous $G_{144}$ selection rules, we found that they are approximately 2 orders of magnitude weaker than the strong pure rotational transitions found in the $A+E$ states. The measurement of these relative intensities is possible because our microwave lines are measured by direct absorption. The relative intensity of our $E+E$ microwave subband may be small partly because the population of the lower state cannot be fully relaxed and also partly because the $E+E$ nuclear-spin statistical weights are smaller. We also note, however, that if $K_{a}$ and $K_{c}$ quantum numbers are assigned to the apparent "asymmetry" doublets (as usual, with $1_{11}$ lower in energy than $1_{10}$, etc.), the observed microwave transitions do not strictly correspond to an $a-, b-$, or $c$-type subband. Rather, the expected rotational selection rules for the $Q$ and $R$ branches of an $a$-type subband are reversed. The observed hyperfine pattern of these transitions is $2-3 \mathrm{MHz}$ wide. When a suitable formalism in $G_{144}$ is derived to interpret the quadrupole hyperfine structure in the ammonia dimer, these well-resolved triplets will undoubtedly provide important structural information.

\section{The $A+E$ states}

The ammonia dimer $A+E$ VRT levels are shown in Fig. 3(b). A total of 392 observed electric dipole transitions connect these states (see Ref. 31). Table II(b) summarizes all the subband origins for the observed transitions connecting the $A+E$ states. The fitted rotational constants, relative $J=0$ energies, and Coriolis coupling constants are shown in Table III(b), and the calculated lowest VRT energy levels $(J=0-5)$ from the fit are listed in Table IV(b).

There are two $G_{1}^{+}$, two $G_{1}^{-}$, two $G_{2}^{+}$, and two $G_{2}^{-}$ tunneling sublevels in $G_{144}$. Pure rotational transitions that do not involve a change in tunneling states are allowed in all of these levels. However, for $\left({ }^{14} \mathrm{NH}_{3}\right)_{2}$, only the $G_{2}^{+}$and $G_{2}^{-}$VRT states have nonzero statistical weight. Before obtaining our new results on the $E+E$ states, we believed the analyses of Nelson et al. ${ }^{8,9}$ and Havenith et al. ${ }^{21}$ that the monomer umbrella inversion tunneling was quenched (i.e., that $G_{36}$ was the appropriate molecular symmetry group), that the interchange tunneling splitting of the $A+E$ states was very small, and that the observed far-infrared transitions involved an $A^{\prime \prime}$ intermolecular vibration. Thus, consideration of the observed $A+E$ states alone would not unambiguously reveal that the correct molecular symmetry group for the ammonia dimer is actually $G_{144}$. As in the $E+E$ states, we must again choose between two possible overall assignments of + and - labels for the $G_{2}$ states in $G_{144}$. We will arbitrarily assume, throughout this paper, that the lowest-energy $K_{a}=0 A+E$ state that we observed has $G_{2}^{+}$symmetry.

Four sets of pure rotational transitions are expected for the $G_{144} A+E$ states, in $K_{a}=0$. Nelson and Klemperer ${ }^{8,9}$ reported only two sets of pure rotational transitions. From far-infrared transitions that we observed, we can deduce that if the state Nelson and Klempercr called $G_{\alpha}$ actually has $G_{2}^{+}$symmetry, then the state they called $G_{\beta}$ really has $G_{2}^{-}$symmetry. These are the two umbrella inversion components of just one of the two $G_{36} G$ states, and they are split $3.3 \mathrm{GHz}$ apart. This partially quenched umbrella inversion frequency is about seven times smaller than that in a free ammonia monomer $(\sim 23.8 \mathrm{GHz}) .^{30}$ Since they are the lowest-energy $A+E$ states that we observed, they most probably correlate to the lower of the two $G_{36} G$ states in the ground vibrational state of the ammonia dimer. The other allowed pair of pure rotational transitions in the next-highest $G_{2}$ states that would correlate to the upper of the two $G_{36} G$ states, was not observed either by us or by Nelson et al. ${ }^{8,9}$ In our microwave search, we were able to observe the pure rotational transitions in the $K_{a}=1$ manifold labeled $(5,6)$ in Fig. 2 , as well as very, very weak pure rotational transitions in the $K_{a}=2$ manifold labeled $(7,8)$. 
These last transitions seemed weak because the $(7,8)$ states, $\sim 500 \mathrm{GHz}$ in energy above the lowest $A+E$ states, should not be well populated in a cold supersonic expansion. However, we could not observe the pure rotational transitions in the $K_{a}=1$ manifold $(3,4)$ which is only $\sim 100 \mathrm{GHz}$ above the lowest $A+E$ states. These observations lead us to believe that the other two $G_{2}$ states (the upper of the two $G_{36} G$ states in the ground vibrational state) are either located more than $500 \mathrm{GHz}$ in energy above the lowest $G_{2}$ states or they have no dipole moment to provide intensity for the pure rotational transitions. We observed very strong $\Delta K_{a}=0$ far-infrared transitions out of all the lower $A+E$ states shown in Fig. 3(b), regardless of whether or not we could observe their pure rotational progressions in the microwave region, and the relative farinfrared intensities seemed to depend only on the relative populations in our supersonic expansion. Therefore, we assume that the population argument applies to the unobserved $G_{2}$ microwave transitions. Later, we deduce that the two $G_{2}$ states in question were found earlier in the farinfrared study by Havenith et al. ${ }^{21}$ near 614 and $617 \mathrm{GHz}$.

The behavior of the $K_{a}=1 A+E$ states is similar to the $E+E$ states that correlate to the $G_{36} E_{1}$ and $E_{2}$ tunneling sublevels. The energy differences between the four $K_{a}=1$ $A+E$ manifolds are much larger than those in $K_{a}=0$. In each $K_{a}=1 A+E$ manifold, the observed splitting of levels with the same $J$ is very small, as is evident in the fitted values of $q B_{n}$ in Table III(b). Coriolis interactions between $K_{a}=0$ and $K_{a}=1$ states cause all the observed values of $q B_{n}$ to be nonzero. However, the analysis presented here explicitly treats only the strongest Coriolis coupling which occurs $\sim 600 \mathrm{GHz}$ above the lowest $A+E$ states. As listed in Table IV(b), the symmetries of the $J=1, K_{a}=1$ states in the affected manifold are $G_{2}^{+}(10)$ and $G_{2}^{-}(9)$. These $1_{1}$ levels are both pushed down by the $G_{2}^{+}(12)$ and $G_{2}^{-}$(11) $1_{0}$ states $\sim 17$ and $\sim 15 \mathrm{GHz}$ higher in energy. Again, this shows that both members of the $K_{a}=1$ pair are symmetric rotational states, $2^{-1 / 2}\left(\left|K_{a}=+1\right\rangle+\left|K_{a}=-1\right\rangle\right)$, that can interact with $K_{a}=0$ rotational states. Also, one state in the pair must be symmetric with respect to umbrella inversion while the other state must be antisymmetric, because the $K_{a}=0$ states they interact with are symmetric and antisymmetric umbrella inversion tunneling states. That is, the umbrella inversion frequency in the $K_{a}=1 A+E$ states is exactly zero. Furthermore, the observed Coriolis interaction between the $K_{a}=1$ manifold $(9,10)$ and the $K_{a}=2$ manifold $(7,8)$, we find that the umbrella inversion frequency in the $K_{a}=2 A+E$ states is also exactly zero.

At this point it becomes possible to assign the two infrared bands observed by Fraser et al. ${ }^{38}$ near $980 \mathrm{~cm}^{-1}$ in double resonance with the lowest $G_{2}$ states. In their modified molecular-beam electric resonance experiment, they measured the strength of the pure rotational $J=1 \leftarrow 0$ transitions in the lowest $G_{2}^{+}$(1) and $G_{2}^{-}$(2) tunneling levels while stepping an overlapping line-tunable $\mathrm{CO}_{2}$ laser between 914 and $987 \mathrm{~cm}^{-1}$. When monitoring the $G_{2}^{+} \mathrm{mi}-$ crowave transition, they observed an infrared peak near $977 \mathrm{~cm}^{-1}$, and when monitoring the $G_{2}^{-}$microwave transition, they observed an infrared peak near $981 \mathrm{~cm}^{-1}$. This approximate $4 \mathrm{~cm}^{-1}$ splitting very likely corresponds to the umbrella inversion tunneling splitting in the upper state. This umbrella inversion frequency is approximately 9 times smaller than that in a free ammonia monomer in $v_{2}=1\left(\sim 36.5 \mathrm{~cm}^{-1}\right),{ }^{30}$ so this result is consistent with our work on the ground state of the ammonia dimer in that both for the ground state and the $v_{2}=1$ state of ammonia the complexed umbrella inversion tunneling frequency is about an order of magnitude smaller than the freeumbrella inversion frequency.

\section{The A+A states}

The four $A+A$ states we have observed are shown in Fig. 3(c). The 89 observed electric dipole transitions that connect these levels are available from PAPS. ${ }^{31}$ The band origins of the three observed subbands are given in Table II(c), the fitted rotational constants and relative $J=0$ energies are shown in Table III(c), and the calculated VRT energy levels $(J=0-5)$ from the fit are listed in Table IV $(\mathrm{c})$

Only one-third of the $G_{144} A+A$ VRT states have nonzero statistical weight for $\left({ }^{14} \mathrm{NH}_{3}\right)_{2}$. It is not possible to distinguish between $G_{36}$ and $G_{144}$ by considering the $A+A$ states alone. The populated $A+A$ tunneling sublevels in $G_{144}$ are $B_{2}^{+}$and $A_{2}^{+}$. The only difference between these and the $G_{36} A_{1}$ and $A_{4}$ states is that the $G_{144}$ states might be slightly shifted by the small umbrella inversion frequency, which we cannot measure. The observed statistical weights show that the lower $A+A$ states have $B_{2}^{+}$tunneling symmetry, which means that they are symmetric interchange tunneling states, while the upper $A+A$ states have $A_{2}^{+}$tunneling symmetry and are thus antisymmetric interchange tunneling states.

No Coriolis perturbations are observed among the $A$ $+A$ states. Yet, the $K_{a}=1 A+A$ states still have nonzero $q B_{n}$ values. Let us assume this $K_{a}=1$ splitting is caused by structural asymmetry, so that we may extend the analysis of Havenith et al. ${ }^{21}$ to extract two pieces of information from the $A+A$ states that we have observed. First, we can confirm that the ammonia dimer molecular plane of symmetry contains the a and $\mathbf{c}$ inertial axes, as we may anticipate by computing the moments of inertia of either the experimental or theoretical structure of the ammonia dimer. Second, we can confirm that the interchange tunneling path is the "trans" path described by Havenith et al. ${ }^{21}$ [by analogy to $(\mathrm{HF})_{2},{ }^{12}$, which has the cyclic $C_{2 h}$ transition state predicted by the $a b$ initio potential-energy surfaces. The other path considered by Havenith et al. ${ }^{21}$ is a "cis" path [with a $C_{2 v}$ transition state, also by analogy to $\left.(\mathrm{HF})_{2}\right]$; cis interchange tunneling results in a different equivalent rotation of the ammonia dimer complex than trans interchange tunneling, and thus different symmetries of the $K_{a}=1$ asymmetry doublets.

To illustrate this, we first assign $K_{a}$ and $K_{c}$ quantum numbers to the observed $A+A$ states. Since there is no way to rotate and displace two ammonia monomers and arrive at a structure in which the $\mathbf{b}$ and $\mathbf{c}$ inertial axes are switched with respect to the starting configuration, we will assume that the $\mathbf{b}$ and $\mathbf{c}$ axes are the same in the upper and 
lower states of the far-infrared transitions. Thus we find, for example, that the $1_{11}$ state in the $K_{a}=1 A+A$ manifold $(2,3)$ has $B_{2}^{+}$symmetry, and the $1_{10}$ state in this manifold has $A_{2}^{-}$symmetry. Recall that the $0_{00}$ state in the $K_{a}=0$ $A+A$ manifold (1) has $B_{2}^{+}$symmetry and that the $1_{01}$ state in the same manifold has $A_{2}^{-}$symmetry. Next, we extend Table II of Havenith et al. ${ }^{21}$ (which lists the symmetry species of the rotational wave functions in $G_{36}$, assuming that the $\mathbf{a}$ and $\mathbf{b}$ inertial axes lie in the plane of symmetry, for the cases of trans interchange tunneling only and cis interchange tunneling only) to the $G_{144}$ molecular symmetry group and to include the possibility that the a and $\mathbf{c}$ inertial axes might lie in the plane of symmetry. In this way, we find that the symmetries of the rotational states that we stated above are only consistent having the $\mathbf{a}$ and $\mathbf{c}$ inertial axes in the plane of symmetry and interchange proceeding along a trans path.

\section{Vibrational assignment}

Several $a b$ initio workers ${ }^{16,18,39}$ have calculated harmonic frequencies for the intermolecular "normal" vibrations of the ammonia dimer. These modes are classified in the $C_{s}$ molecular symmetry group, but the grouptheoretical correlation tables presented earlier in this paper can be used to enumerate all the $G_{144}$ tunneling sublevels that correlate to each of these $C_{s}$ rovibrational states. The specific $G_{144}$ symmetry of each vibration can be multiplied by the symmetries of the ground-state tunneling sublevels as an initial estimate of the ordering of the tunneling sublevels in the excited vibrational state. We have argued above that all of the lower states of the far-infrared subbands are symmetric with respect to the donor-acceptor interchange tunneling and all of the upper states are correspondingly antisymmetric. We now address the following question: Do the far-infrared transitions connect the symmetric and antisymmetric interchange levels within the ground vibrational state of the ammonia dimer complex, or does the upper state correspond to a low-frequency intermolecular vibration?

We have no data that rule out the first possibility. Hassett, Marsden, and Smith $^{20}$ and Frisch et al. ${ }^{16}$ calculate a low barrier to interchange tunneling. That is, they find that the cyclic transition state is only $0.1-0.2 \mathrm{kcal} / \mathrm{mol}$ above their calculated equilibrium structure. In addition, they find that the harmonic zero-point energy of the vibration in the same coordinate as the lowest-energy path for the interchange tunneling motion is approximately equal to the barrier height. Our observation of a large interchange tunneling splitting is consistent with this feature of highquality theoretical surfaces.

Frisch et al., ${ }^{16}$ Andrews and Dykstra, ${ }^{18}$ and Hassett, Marsden, and Smith ${ }^{39}$ all predict a very-low frequency torsional mode with a harmonic frequency at 20,27 , and 35 $\mathrm{cm}^{-1}$, respectively. All three groups give the $C_{s}$ symmetry of this mode as $A^{\prime \prime}$. Assuming that the intermolecular vibrations of the ammonia dimer have one dimensional representations in $G_{144}$, the $G_{144}$ symmetry of this torsional mode is $A_{1}^{-}, B_{1}^{-}, A_{2}^{-}$, or $B_{2}^{-}$. Our observations rule out each of these possibilities. The upper state of our far. infrared transitions have a vibrational symmetry of either $A_{1}^{+}, B_{1}^{+}, A_{2}^{+}$, or $B_{2}^{+}$and correlates to a $C_{s}$ mode with $A^{\prime}$ symmetry. That is, the $K_{a}=0 \leftarrow 0$ transitions in the $A+A$ states can only be observed if the vibrational symmetry has a + label, the fact the two close $K_{a}=0 E+E$ tunneling levels that have the same symmetry have + labels in both the lower and upper states also requires that the vibrational symmetry have a + label, and the fact that we always observe the $K_{a}=0 G_{2}^{+}$level below the $G_{2}^{-}$level also suggests that the vibrational symmetry has a + label. The lowest-energy $A^{\prime}$ mode predicted by the various theoretical workers lies at 121,81 , and $72 \mathrm{~cm}^{-1}$, respectively, and is the vibration along the interchange tunneling coordinate we mentioned above. We do not believe that the assignment of our spectra to this vibration is reasonable since our transitions occur at a factor of 4 lower in energy, and because we have not assigned any subbands that might originate from the antisymmetric interchange levels of the ground state in the event that we were observing this vibrational band.

\section{DISCUSSION}

In this paper, we have presented extensive new information on the VRT levels of the ammonia dimer. The charactcrization of all the ground-state tunnelingrotational energy levels in $K_{a}=0$ and $K_{a}=1$ is complete. We have definitively assigned all previously reported farinfrared subbands ${ }^{21,23}$ (the new assignments are noted in Table II), and indeed, the information provided by any new measurements of far-infrared subbands within the ground state of the ammonia dimer will be redundant with the results presented here. There should be a few strong far-infrared subbands at lower frequency from where we scanned: in the $A+A$ states, there should be a $K_{a}=0 \leftarrow 1$ subband at $272.8 \mathrm{GHz}[(4) \leftarrow(2,3)]$; in the $A+E$ states, there should be $K_{a}=0 \leftarrow 1$ subbands at 382.6 and 385.0 $\mathrm{GHz}[(11) \leftarrow(5,6)$ and $(12) \leftarrow(5,6)]$; and in the $E+E$ states, there should be $K_{\alpha}=1 \leftarrow 0$ subbands at $373.6,374.6$, and $376.0 \mathrm{GHz}$ (this last one might be weak) $[(17,18) \leftarrow(4),(17,18) \leftarrow(3)$, and $(17,18) \leftarrow(1)]$. The frequencies of the lowest $J$ transitions in these subbands can be calculated to within $1 \mathrm{MHz}$ from Table IV. The last five subbands involve upper or lower states that are perturbed by Coriolis interactions, and in $\Delta K_{a}= \pm 1$ subbands involving these perturbed states we have noticed intensity perturbations that made the $R$ branch particularly strong and the $P$ branch very weak (or vice versa).

Exploiting our new definitive characterization of the ground vibrational state of the ammonia dimer, we are able to improve on the interpretation of the infrared spectrum of the ammonia dimer measured in the monomer $v_{2}=1$ region by Huisken and Pertsch ${ }^{40}$ and by Snels et al. ${ }^{41}$ Since line-tunable $\mathrm{CO}_{2}$ lasers were used in these experiments, and since the upper state of these infrared spectra dissociates rather quickly, rotational resolution of these bands was not possible. The main features found in these spectra are a broader, more intense band, with obvious, but still unresolved structure near $979 \mathrm{~cm}^{-1}$, and a sharper single band near $1004 \mathrm{~cm}^{-1}$. These two bands were interpreted as 
excitations of the two inequivalent ammonia subunits within the complex. However, we now know that it is not possible to excite either the donor or the acceptor by itself when donor-acceptor interchange tunneling is occurring within the complex. van Bladel et al. show in the accompanying paper that only the $A+E$ states are "localized" with respect to interchange tunneling. Thus, it may be useful to think of this entire spectrum as components of a single transition from the ground vibrational state, that we have characterized here, to a single excited state. In the $K_{a}=0 \leftarrow 0$ portion of this spectrum, four subbands involving the $A+E$ states should be observable: two from the lower $A+E$ states found by Nelson et al. ${ }^{8,9}$ to the lower $A+E$ states in the excited vibrational states, and two from the lower $A+E$ states to the upper $A+E$ states in the excited vibrational state. Both pairs are expected to be strong transitions because the pure rotational transitions in the lower $A+E$ states in the ground state and the transitions across the $A+E$ tunneling splitting in the ground state are both strong. In their infrared-microwave doubleresonance experiment, Fraser et $a l .^{38}$ identified one pair of $A+E$ transitions under the $979 \mathrm{~cm}^{-1}$ peak, but did not scan under the $1004 \mathrm{~cm}^{-1}$ peak, because the normal isotope $\mathrm{CO}_{2}$ laser that they were using does not provide that frequency. We believe that the second pair of $A+E$ transitions lies under the $1004 \mathrm{~cm}^{-1}$ infrared peak. This $\sim 25$ $\mathrm{cm}^{-1}$ internal rotation and interchange tunneling splitting of the $A+E$ states in $v_{2}=1$ is not very different from the value that we have measured in the ground state.

It had previously been thought that the anisotropy of the intermolecular potential completely "quenched" the umbrella inversion of the ammonia monomers, but our results clearly show that this motion still occurs in the ammonia dimer complex. This leads to the molecular symmetry group $G_{144}$, rather than the group $G_{36}$, which has guided earlier analyses. In this work, we have identified $K_{a}=0$ umbrella inversion tunneling splittings of 3.3094(3) $\mathrm{GHz}$ and 2.3920 (6) $\mathrm{GHz}$ in the "lower" and "upper" $A$ $+E$ states, respectively. In the $E+E$ states, the umbrella inversion tunneling splittings are $1.094(1) \mathrm{GHz}$ (splitting of the $G_{30} E_{3}$ level), $1.103(1) \mathrm{GHz}\left(E_{1}\right), 2.217(1) \mathrm{GHz}$ $\left(E_{4}\right)$, and $2.217(1) \mathrm{GHz}\left(E_{2}\right)$. These frequencies, are about 10 times less than the inversion tunneling splitting of $\sim 23.8 \mathrm{GHz}$ (Ref. 30) in the ground state of the free ammonia monomer. Thus, it is indeed true that monomer umbrella inversion tunneling is partially quenched in the ammonia dimer; it occurs on essentially the same time scale as end-over-end rotation in the low- $J, K_{a}=0$ states.

In $\mathrm{Ar}-\mathrm{NH}_{3}$, the umbrella inversion of the monomer is nearly free in the observed $|\Omega|=0$ states, but completely quenched in the observed $|\Omega|=1$ states, in which the ammonia lone pair tends to point either towards or away from the argon atom. ${ }^{36,37}$ However, the two $|\Omega|=1 E$ states of $\mathrm{Ar}-\mathrm{NH}_{3}$ that correlate to the $j=1,\left|k_{c}\right|=1$ states of the ammonia monomer are split apart by $200 \mathrm{GHz} .{ }^{29}$ This splitting would occur in the absence of monomer inversion and it arises largely because there is an energy difference between the localized ammonia-lone-pair-towards-argon and tions. ${ }^{36,37}$ These $|\Omega|=1 \mathrm{Ar}-\mathrm{NH}_{3} E$ states show no true asymmetry doubling. However, small splittings (that look like "asymmetry doubling") are observed because each initially degenerate component of the $|\Omega|=1 E$ states is Coriolis coupled to a different $|\Omega|=0$ state, and these two nearby $|\Omega|=0$ states are split $\sim 22.7 \mathrm{GHz}$ apart by monomer umbrella inversion. ${ }^{29}$ In the ammonia dimer, the $K_{a}$ $=1 A+E$ states and the $K_{a}=1 E+E$ states that correlate to the $G_{36} E_{1}$ and $E_{2}$ states behave like the $|\Omega|=1 E$ states of $\mathrm{Ar}-\mathrm{NH}_{3}$. The umbrella inversion in all of these ammonia dimer $K_{a}=1$ states is quenched and they show no true asymmetry doubling, as explained in the preceding sections. The $K_{a}=1$ separations of these states (large energy separations which are not caused by monomer inversion) are $118.0887(7)$ and $378.4596(6) \mathrm{GHz}$ in the lower and upper $A+E$ states, respectively, and $366.694(1)$ and 678.199 (1) $\mathrm{GHz}$ in the $E+E$ states that correlate to the $G_{36}$ states $E_{1}$ and $E_{2}$, respectively. It is interesting to note that, by looking at the $G_{144}$ symmetry labels alone, we cannot tell the difference between states that are members of an asymmetry doublet and states that are members of umbrella inversion doublets in the ammonia dimer $A+E$ states, in the ammonia dimer $E+E$ states that correlate to the $G_{36} E_{1}$ and $E_{2}$ states, or in the $\mathrm{Ar}-\mathrm{NH}_{3} E$ states. By contrast, the ammonia dimer $K_{a}=1$ states that correlate to $G_{36} E_{3}$ and $E_{4}$ states show both real asymmetry doubling and monomer umbrella inversion tunneling splittings. In these states, the $G_{144}$ symmetry labels do distinguish the asymmetry doublets from the umbrella inversion tunneling doublets. For example, the asymmetry doublets in one umbrella inversion state are $G_{4}^{+}\left(E_{3}\right)$ and $G_{3}^{-}\left(E_{4}\right)$, while in the other umbrella inversion state they are $G_{4}^{-}\left(E_{3}\right)$ and $G_{3}^{+}\left(E_{4}\right)$. In these $K_{a}=1$ states we have measured the very small umbrella inversion tunneling splittings of $48(1)$ $\mathrm{MHz}$ of the lower $E_{3}$ state ( $G_{36}$ tunneling symmetry) and 9 (1) $\mathrm{MHz}$ of the upper $E_{4}$ state ( $G_{36}$ tunneling symmetry).

In the ammonia dimer, we have identified donoracceptor interchange tunneling frequencies of 483.2995(4) $\mathrm{GHz}\left(K_{a}=0\right)$ and $518.5007(6) \mathrm{GHz}\left(K_{a}=1\right)$ in the $A+A$ states, $578.4255(9) \mathrm{GHz}\left(K_{a}=0\right)$ and $730.8030(7) \mathrm{GHz}$ ( $\left.K_{a}=1\right)$ between the $E+E$ states that correlate to the $G_{36}$ $E_{3}$ and $E_{4}$ states, and $580.8738(7) \mathrm{GHz}\left(K_{a}=0\right)$ and 609.3528 (7) $\mathrm{GHz}\left(K_{a}=1\right)$ between the $E+E$ states that correlate to the $G_{36} E_{1}$ and $E_{2}$ states. The corresponding measured frequencies in the $A+E$ states are 614.1768(3) $\mathrm{GHz}\left(K_{a}=0\right)$ and $616.9779(4) \mathrm{GHz}\left(K_{a}=1\right)$, but this splitting is due to a difference in donor and acceptor internal rotation frequencies that has been increased due to interchange tunneling (i.e., interchange has a second-order effect on this splitting of the $A+E$ states). Because of their initial energy separation caused by internal rotation, the interchange tunneling does not mix the lower and upper $A+E$ states very much, so that they cannot be called symmetric or antisymmetric with respect to interchange tunneling. This is clearly illustrated in the lowest $A+E$ wave functions by van Bladel et $a l^{22}$ in the accompanying paper. Let us assume the upper limit of $400 \mathrm{GHz}$ for the initial internal rotation separation of these $A+E$ states, which 
corresponds to free internal rotation of the acceptor about its $C_{3}$ axis and no internal rotation of the donor. Let us further assume that interchange tunneling mixes only the lower and upper $A+E$ states that belong to the ground vibrational state of the complex; this is probably the most important effect of interchange feasibility on the $A+E$ states. Then, we can solve for the off-diagonal "interchange coupling" of the $A+E$ states in this two-state approximation. We find that the interchange matrix element is $\sim 230$ $\mathrm{GHz}$ for the $A+E$ states. Thus, if there were initially zero internal rotor splitting (rather that the $400 \mathrm{GHz}$ we assumed) between these $A+E$ states, interchange tunneling would split them by $\sim 460 \mathrm{GHz}$. Since we assumed the upper limit for the initial internal rotor splitting, $460 \mathrm{GHz}$ is a lower limit for the interchange tunneling frequency in the two-state approximation. The upper limit for the interchange tunneling frequency in the $A+E$ states is the measured $\sim 615 \mathrm{GHz}$ splitting.

Donor-acceptor interchange tunneling frequencies have also been measured in other hydrogen-bonded homodimers, namely $(\mathrm{HCl})_{2},(\mathrm{HF})_{2}$, and $\left(\mathrm{H}_{2} \mathrm{O}\right)_{2}$. For $\left(\mathrm{H}^{35} \mathrm{Cl}\right)_{2}$, the donor-acceptor interchange tunneling splitting has been measured at $463.979(2) \mathrm{GHz}$ (Ref. 13) by tunable far-infrared laser spectroscopy, and the barrier to this motion has been empirically estimated to be 28.4 $\mathrm{cm}^{-1.42}$ For $(\mathrm{HF})_{2}$, the donor-acceptor interchange tunneling splitting has been measured in the microwave at $19.94704(2) \mathrm{GHz}^{43}$ and an empirical potential-energy surface puts this barrier at $302 \mathrm{~cm}^{-1} \cdot{ }^{44}$ And for $\left(\mathrm{H}_{2} \mathrm{O}\right)_{2}$, the donor-acceptor interchange tunneling splitting has also been measured in the microwave at $19.52673 \mathrm{MHz}^{14}$ and the $a b$ initio barrier to this motion is $304 \mathrm{~cm}^{-1}$. 45 The interchange tunneling frequency in the ammonia dimer $A$ $+A$ states $(483.2 \mathrm{GHz})$ is quite similar to the interchange tunneling frequency in $(\mathrm{HCl})_{2}(464.0 \mathrm{GHz})$. In both complexes, the interchange tunneling motion is predicted to occur via rotation of both monomers, each about an axis perpendicular to the plane of the dimer, so that we can compare the reduced masses for interchange tunneling by comparing the monomer rotational constants. For the ammonia monomer, $A=B \approx 9.9 \mathrm{~cm}^{-1}$ and for $H^{35} \mathrm{Cl}$ monomer, $B \approx 10.6 \mathrm{~cm}^{-1} ; 46$ these values only differ by $\sim 7 \%$. Recently, Hassett, Marsden, and Smith ${ }^{20}$ have calculated a barrier height of $29 \mathrm{~cm}^{-1}$ for donor-acceptor interchange tunneling in the ammonia dimer. Since both the interchange tunneling frequencies and the reduced masses for this motion are similar for the ammonia dimer and the hydrogen chloride dimer, we believe that our measurements support the $29 \mathrm{~cm}^{-1}$ barrier predicted by Hassett, Marsden, and Smith, which is also similar to the empirical barrier of $28.4 \mathrm{~cm}^{-1}$ deduced for $(\mathrm{HCl})_{2}$. By contrast, the rotational constant for $(\mathrm{HF})_{2}$ is $\approx 20.9 \mathrm{~cm}^{-1}$. ${ }^{46}$ If the barrier to interchange tunneling in the ammonia dimer were at all similar to the $\sim 300 \mathrm{~cm}^{-1}$ barrier to interchange tunneling in the hydrogen fluoride dimer, the interchange frequency in the ammonia dimer should be less than the $\sim 20$ $\mathrm{GHz}$ interchange frequency in the hydrogen fluoride dimer. This is clearly far from being the case.

Some time ago, Frisch et al. ${ }^{16}$ also calculated the bar- rier to interchange tunneling in the ammonia dimer, and arrived at a value of $\sim 70 \mathrm{~cm}^{-1}$. A similar value of 77 $\mathrm{cm}^{-1}$ is computed by van Bladel et al. ${ }^{22}$ for the site-site potential of Sagarik, Ahlrichs, and Brode. ${ }^{19}$ In the accompanying paper, van Bladel $e t$ al. ${ }^{22}$ show that a surface with a barrier of $167 \mathrm{~cm}^{-1}$ gives an interchange tunneling frequency that is three times lower than what is observed. Liu et $a{ }^{18}{ }^{18}$ have performed a high-level calculation of the electrostatic interaction surface for the ammonia dimer at fixed $R_{\mathrm{CM}}$ (including electric multipole-multipole interactions, induction, dispersion, but no short-range repulsion) and obtained an electrostatic contribution to this barrier of 250 $\mathrm{cm}^{-1}$. This reveals that the $a b$ initio values for this interchange barrier are the sums of large opposing contributions, so that this feature of the ammonia dimer potentialcnergy surface is the most difficult to predict accurately.

The "local internal axis method (IAM)," a model developed by Hougen which assumes high barriers to tunneling, is a type of analysis that does not require knowledge of the entire potential-energy surface. This approach has been used quite fruitfully to characterize the tunneling motions and some of the energetics of (HF) $)_{2}$ (Ref. 12) and $\left(\mathrm{H}_{2} \mathrm{O}\right)_{2}{ }^{15}$ In such a high barrier limit, Coudert and Hougen $^{35}$ have derived possible tunneling energy-level patterns for $\left(\mathrm{XY}_{3}\right)_{2}$ molecules in the $G_{36}$ molecular symmetry group. A general result of their work is that the interchange tunneling splitting of the $A+A$ states must be larger than the interchange tunneling splitting of the $E+E$ states. This is because the splitting of the $A+A$ states involves the sum of all the various contributions from different possible tunneling paths, while the splitting of the $E$ $+E$ states involves both sums and differences of the same contributions. In this work, we find that the $E+E$ interchange frequency of the ammonia dimer is much larger than the $A+A$ interchange frequency (by $100 \mathrm{GHz}$ ). This is more than can possibly be accounted for by the umbrella inversion tunneling not considered by Coudert and Hougen. The authors do mention that they suspect the high barrier analysis will not be appropriate for the ammonia dimer, and they are indeed correct.

Both Nelson et al. ${ }^{9}$ and Havenith et al. ${ }^{21}$ have argued that the internal rotation tunneling frequency is much faster than the interchange tunneling frequency in the ammonia dimer. That is, they believed that the $2 C_{3}+I$ limit proposed by Nelson and Klemperer ${ }^{10}$ was an appropriate description of tunneling dynamics in this complex. Although nuclear-spin statistics prevent us from directly measuring the internal rotation tunneling frequency, we can use the measured rotational constants for the $A+A$, $A+E$, and $E+E$ states to compare the internal rotation tunneling frequency to the other known tunneling frequencies. The $K_{a}=0$ tunneling states that are separated by only umbrella inversion tunneling frequencies have nearly identical $B_{n}$ rotational constants, which deviate from each other by less than $0.02 \%$. [These are the $A+E$ states (1) $G_{2}^{+}$and (2) $G_{2}^{-}, A+E$ states (11) $G_{2}^{+}$and (12) $G_{2}^{-}, E+E$ states (1) $G_{4}^{+}\left(E_{3}\right)$ and (2) $G_{4}^{-}\left(E_{3}\right), E+E$ states $(3) G_{3}^{-}$ $\left(E_{1}\right)$ and $(4) G_{4}^{+}\left(E_{1}\right), E+E$ states $(13) G_{3}^{+}\left(E_{4}\right)$ and (14) $G_{3}^{-}\left(E_{4}\right)$, and $E+E$ states $(15) G_{4}^{-}\left(E_{2}\right)$ and (16) 
$G_{3}^{+}\left(E_{2}\right)$.] On the other hand, the $K_{a}=0$ tunneling states that are separated by the interchange tunneling frequency have $B_{n}$ values that deviate from each other by $1 \%-2 \%$. [These are the $A+A$ states (1) $B_{2}^{+}$and (4) $A_{2}^{+}$, the $A+E$ states (1) and (11) both $G_{2}^{+}$, the $A+E$ states (2) and (12) both $G_{2}^{-}$, the $E+E$ states (1) $G_{4}^{+}\left(E_{3}\right)$ and (13) $G_{3}^{+}\left(E_{4}\right)$, the $E+E$ states (2) $G_{4}^{-}\left(E_{3}\right)$ and (14) $G_{3}^{-}\left(E_{4}\right)$, the $E+E$ states (3) $G_{3}^{-}\left(E_{1}\right)$ and (15) $G_{4}^{-}\left(E_{2}\right)$, and the $E+E$ states (4) $G_{4}^{+}\left(E_{1}\right)$ and (16) $G_{3}^{+}\left(E_{2}\right)$.] The $B_{n}$ rotational constants of the $K_{a}=0$ tunneling states that are separated by only the internal rotation tunneling frequency deviate from each other by $0.3 \%-0.4 \%$. [These are taken as the average $B_{n}$ values for the $A+A, A+E$, and $E+E$ states that correlate to the lower (symmetric) interchange tunneling state and the average $B_{n}$ values for the $A+A, A+E$, and $E+E$ states that correlate to the upper (antisymmetric) interchange tunneling state.] When compared to the other two observed deviations in rotational constants, this implies that the internal rotation tunneling frequency is smaller that the interchange tunneling frequency, but larger than the umbrella inversion frequency; it is probably several wave numbers in magnitude. We emphasize, however, that the internal rotation frequency is not very small. The strong Coriolis coupling observed between manifolds with different $K_{a}$ is evidence that the tunneling sublevels of the ammonia dimer do have some substantial internal angular momentum. The absolute upper limit for the internal rotation frequency would be $400 \mathrm{GHz}$ or $13.3 \mathrm{~cm}^{-1}$, the rotational energy of a free ammonia monomer in the $1_{1}$ rotational state. However, even this upper limit is smaller than the observed donor-acceptor interchange tunneling frequencies. In other words, our observations lead us to conclude that the opposite $I+2 C_{3}[+i]$ limit proposed by Nelson and Klemperer ${ }^{10}$ is the appropriate description of the tunneling motions in the ammonia dimer-not the $2 C_{3}$ $+I$ limit that has becn assumed in previous analyses.

We can also compare the observed $A$ rotational constants of the ammonia dimer to the $A$ rotational constant predicted from the structure of Nelson et al. ${ }^{8,9}(115 \mathrm{GHz})$ and from a linearly hydrogen-bonded structure (105 $\mathrm{GHz}$ ). The average energy difference between the $K_{a}=0$ and $K_{a}=1$ levels $(\approx A$ ) of the $A+A$ states is $228 \mathrm{GHz}$, for the $A+E$ states it is $173 \mathrm{GHz}$, and for the $E+E$ states it is $111 \mathrm{GHz}$. These values are closer to the predicted value for rotation of the entire ammonia dimer about its a inertial axis than to the value $(400 \mathrm{GHz})$ for rotation of an ammonia monomer constituent about its $\mathrm{c}$ axis. By contrast, in the ammonia water complex, in which internal rotation of the ammonia monomer about its $C_{3}$ axis is nearly free, the structural value of $A$ is $\sim 150 \mathrm{GHz}$, but $K_{a}=1 \leftarrow 0$ transitions in the ammonia water $A$ states were measured near $620 \mathrm{GHz} .{ }^{34}$ This comparison suggests that the internal rotation cannot be as free in the ammonia dimer as it is in the ammonia water complex. In the ammonia dimer, the internal rotation frequency must be smaller than the interchange frequency in order for the interchange tunneling motion to average out the effect of acceptor internal rotation on the $A$ rotational constant.

Liu $e t a l .{ }^{18}$ have also calculated the electrical interac- tion contributions to the internal rotation barriers in the ammonia dimer. They find, at a hydrogen-bonded configuration with $\theta_{A}=12.8^{\circ}$ and $\theta_{B}=102^{\circ}$ in Fig. 1 , that the barrier to accepter internal rotation is $\approx 10 \mathrm{~cm}^{-1}$, while the barrier to donor internal rotation is $\approx 650 \mathrm{~cm}^{-1}$. At the cyclic transition state, with $\theta_{A}=\theta_{B}=62.5^{\circ}$, the internal rotation barrier is $\approx 210 \mathrm{~cm}^{-1}$. This suggests that there may be a considerable "interchange averaged" barrier to internal rotation in the ammonia dimer. However, these calculations did not include the possible effects of shortrange forces, which could substantially change these barriers.

Finally, we turn to the structure of the ammonia dimer. In this paper, we have clearly demonstrated that a substantial amount of large-amplitude tunneling motion occurs on the time scale of our experiment. It makes little sense to speak of a rigid structure per se for the ammonia dimer, and it is particularly difficult to imagine the monomer inversion occurring in a rigid configuration. Thus, when we discuss the "structure" of the ammonia dimer, we refer to the minima of its intermolecular potential-energy surface, rather than to fixed positions of the constituent nuclei.

The structure proposed by Nelson et al. ${ }^{8,9}$ was based on the assumption that neither the donor-acceptor tunneling nor zero-point vibrational averaging affects the measured ${ }^{14} \mathrm{~N}$ quadrupole coupling constants in the two observed $K_{a}=0 A+E$ pure rotational progressions. In the accompanying paper, van Bladel et al. ${ }^{22}$ show that it is indeed true that these $A+E$ wave functions are "localized" with respect to the interchange tunneling. This same qualitatively localized behavior is also characteristic of the water dimer $E$ states, which are comprised of one ortho water monomer plus one para water monomer. ${ }^{14}$ This effect is unique to homodimers involving two monomers in different proton nuclear-spin states. By contrast, such "localization" is not expected for any of the tunneling states of $(\mathrm{HF})_{2}$ (Refs. 11 and 12) or $(\mathrm{HCl})_{2}$-and, indeed, only one average ${ }^{35} \mathrm{Cl}$ or ${ }^{37} \mathrm{Cl}$ quadrupole coupling constant is measured in $\left(\mathrm{H}^{35} \mathrm{Cl}\right)_{2}$ or $\left(\mathrm{H}^{37} \mathrm{Cl}\right)_{2}{ }^{13}$ Further examples are the $A$ and $B$ states of $\left(\mathrm{H}_{2} \mathrm{O}\right)_{2},{ }^{14}$ and the $A+A$ and $E+E$ states of $\left(\mathrm{NH}_{3}\right)_{2}{ }^{22}$ Similar to the case of the $E$ states of the water dimer, van Bladel et al. ${ }^{22}$ find that the localized $A+E$ states of the ammonia dimer do not necessarily have to be close in energy, contrary to the stated expectations of Nelson et al. ${ }^{9,10}$ This finding is confirmed by our experimental results. van Bladel et al. ${ }^{22}$ also find that the $a b$ initio surface used in their quantum dynamical calculations is sufficiently flat that vibrational averaging causes the measured values of $\left.e q Q_{a a}\left(\mathrm{NH}_{3}^{A}\right)\left(\left\langle P_{2}(\cos ) \theta_{A}\right\rangle\right)\right)$ and $e q Q_{a a}\left(\mathrm{NH}_{3}^{B}\right)\left(\left\langle P_{2}\left(\cos \theta_{B}\right)\right\rangle\right)$ to yield different values of $\theta_{A}$ and $\theta_{B}$ than those that seem consistent with the measured value of

$$
\mu_{a} \propto\left(\left\langle P_{1}\left(\cos \theta_{A}\right)\right\rangle+\left\langle P_{1}\left(\cos \theta_{B}\right)\right\rangle\right),
$$

such that all these observed quantities cannot be used together to deduce the structure, as Nelson, Fraser, and Klemperer ${ }^{8}$ have done. In addition, van Bladel et $a l^{22}$ find that both of the above methods of estimating $\theta_{A}$ and $\theta_{B}$ 
yield values that deviate significantly from the equilibrium values of $\theta_{A}$ and $\theta_{B}$ for the surface used. van Bladel et al. ${ }^{22}$ are the first workers to actually quantify these deviations, thus removing all doubt that the effects of vibrational averaging must be considered in the determination of the ammonia dimer equilibrium structure.

It is now obvious that an appropriate analysis has not yet been performed to determine the equilibrium structure of the ammonia dimer. The proper way to approach this problem, which is beyond the scope of the present paper, is to fit a full six-dimensional (or eight-dimensional, if it is necessary to treat the monomer umbrella inversion in full detail) potential-energy surface to all of the spectroscopic data presented here, and then find the most-stable structure described by this fitted potential. This method would involve multiple iterations of the VRT state calculations described by van Bladel et al. as required by a nonlinear least-squares-fitting routine, and this would be computationally very demanding. Such analyses have recently yielded experimental potential-energy surfaces for $\mathrm{Ar}-\mathrm{H}_{2} \mathrm{O}$ (three dimensions), ${ }^{1,47}$ and for $\mathrm{Ar}-\mathrm{NH}_{3}$ (Ref. 37) (three dimensions plus an adiabatic treatment of monomer inversion), using the very efficient collocation method to calculate the VRT states of these complexes.

Theoretical investigators ${ }^{16-20}$ who calculated $a b$ initio intermolecular potential-energy surfaces for the ammonia dimer immediately following the experiments of Nelson $e t$ $a l^{8,9}$ also suggest that "vibrational averaging" on their surfaces would lead to the experimentally observed molecular constants. Since we no longer need to judge theoretical surfaces by the criterion that they possess equilibrium structures that match the vibrationally averaged structure proposed by Nelson et al., we now reexplore the proposed $a b$ initio structures. In general, the workers who used the largest basis sets, optimized all degrees of freedom, and accounted for electron correlation and basis-set superposition error, found that a nearly linearly hydrogen-bonded geometry was the equilibrium configuration for the ammonia dimer. ${ }^{16,20}$ In the coordinates of Fig. 1, Hassett et al. ${ }^{16}$ found $\theta_{A} \approx 24^{\circ}$ and $\theta_{B} \approx 94^{\circ}$, with $\phi_{A}$ rotated by $60^{\circ}$ from the way we have drawn it. Frisch et al. ${ }^{16}$ found $\theta_{A} \approx 8^{\circ}$ and $\theta_{B} \approx 110^{\circ}$. Most the experimental information that has been directly compared with the $a b$ initio results comes from the Klemperer laboratory. The theoretically predicted linearly hydrogen-bonded structure has a dipole moment of two dimensions. ${ }^{16,18,20}$ Vibrational averaging along the interchange tunneling coordinate could make the measured dipole moment smaller than two dimensions, and should affect the normal isotopomer more than the fully deuterated isotopomer. However, Nelson et al. ${ }^{8,9}$ found that the measured dipole moment of $0.5 \mathrm{D}$ for $\left(\mathrm{ND}_{3}\right)_{2}$ is actually smaller than the measured dipole moment of $0.8 \mathrm{D}$ for $\left(\mathrm{NH}_{3}\right)_{2}$. This result was thought to rule out the linearly hydrogen-bonded structure. Recently, however, Hassett, Marsden, and Smith ${ }^{20}$ have finally pointed out the plausible explanation for the measured dipole moments consistent with their $a b$ initio surface. Several competing effects are involved: while the zero-point vibration in the interchange tunneling coordinate leads toward a cyclic geome- try which has no dipole moment, thus predicting a smaller expected dipole moment for $\left(\mathrm{NH}_{3}\right)_{2}$, zero-point motion in the orthogonal in-plane vibrational mode leads to a headto-tail geometry which has an approximately threedimensional dipole moment, thus predicting a larger dipole moment for $\left(\mathrm{NH}_{3}\right)_{2}$ than for $\left(\mathrm{ND}_{3}\right)_{2}$.

Another calculation that favors the linearly hydrogenbonded structure is the empirical study by Liu et al. ${ }^{18}$ They computed highly accurate electrostatic contributions to the angular potential-energy interaction between two ammonia monomers fixed at the experimental center of mass distance. They, too, arrived at a configuration with $\theta_{A}=12^{\circ}$ and $\theta_{B}=101^{\circ}$. As we mentioned earlier, this calculation resulted in higher barriers to the various tunneling motions than the full $a b$ initio potentials predict. Dykstra and Andrews $^{18}$ later found that it is possible to fit atom-atom repulsive contributions to augment the electrical interaction surface that reduce the interchange barrier and can even shift the equilibrium structure towards the structure proposed by Nelson et $a l^{8,9}$

Other recent $a b$ initio structural calculations which do not have a linearly hydrogen-bonded equilibrium structure are less convincing. Latajka and Scheiner ${ }^{17}$ used a smaller basis than either Frisch et al. ${ }^{16}$ or Hassett, Marsden, and Smith, ${ }^{20}$ and found that a cyclic geometry with $\theta_{A}=68^{\circ}$ and $\theta_{B}=112^{\circ}$, which is close to the transition state of Frisch et al. and Hassett, Marsden, and Smith, was the equilibrium structure on their surface, and is $0.2 \mathrm{kcal} / \mathrm{mol}$ more stable than the linearly hydrogen-bonded form. Hassett, Marsden, and Smith ${ }^{20}$ were able to obtain similar results when they reduced their basis size. Such an equilibrium structure is definitively ruled out by our experimental data. All of the electric-dipole-allowed transitions that are required to involve a change of interchange tunneling state in the $C_{s}$ conformer would become pure rotational transitions in this proposed $C_{2 h}$ conformer, and it is unclear how such a structure can have a dipole moment, which is clearly required for such transitions to be observable. Sagarik, Ahlrichs, and Brode ${ }^{19}$ claim that they have predicted an equilibrium structure $\left(\theta_{A}=61^{\circ}\right.$ and $\left.\theta_{B}=105^{\circ}\right)$ very similar to the one proposed by Nelson et al. However, van Bladel et al. ${ }^{22}$ have shown that the true minimum of that particular surface actually occurs at the linearly hydrogen-bonded geometry! The proposed structure of Sagarik, Ahlrichs, and Brode ${ }^{19}$ was originally preferred by Nelson et al. ${ }^{9}$ because a calculation of vibrational averaging effects was not necessary in order to achieve agreement between the theoretically and experimentally proposed structures.

\section{ACKNOWLEDGMENTS}

We thank Dr. Luca Dore, Nick Pugliano, and Jeff Cruzan for their help with the far-infrared search, and Sakae Suzuki and Paul Stockman for their help with the microwave experiments. We thank Professor Ad van der Avoird, Nick Pugliano, Paul Stockman, and Sakae Suzuki for providing useful comments on this manuscript. J.G.L. would like to thank Joyce Bumgarner for three weeks of hospitality in Pasadena, CA, while most of the microwave 
experiments were performed. This work was supported by the National Science Foundation, Grant No. CHE8612296. G.A.B. would like to thank the David and Lucille Packard and Alfred P. Sloan Foundations for support.

'R. C. Cohen and R. J. Saykally, Annu. Rev. Phys. Chem. 42, 369 (1991): J. Phys. Chem. 96, 1024 (1992).

2D. J. Nesbitt, Chem. Rev. 88, 843 (1988).

${ }^{3}$ A. C. Legon, Chem. Soc. Rev. 19, 197 (1990).

4A. D. Buckingham, P. W. Fowler, and J. M. Hutson, Chem. Rev. 88, 963 (1988).

${ }^{5}$ G. C. Pimentel, M. O. Bulanin, and M. van Thiel, J. Chem. Phys. 36, 500 (1962).

${ }^{\circ} \mathrm{A}$ list of references in which the ammonia dimer is assumed to be a hydrogen-bonded system is given in the introduction of Ref. 17 .

'T. R. Dyke, K. M. Mack, and J. S. Muenter, J. Chem. Phys. 66, 498 (1977).

${ }^{8}$ D. D. Nelson, G. T. Fraser, and W. Klemperer, J. Chem. Phys. 83, 6201 (1985).

${ }^{9}$ D. D. Nelson, W. Klemperer, G. T. Fraser, F. J. Lovas, and R. D. Suenram, J. Chem. Phys. 87, 6364 (1987).

${ }^{10}$ D. D. Nelson and W. Klemperer, J. Chem. Phys. 87, 139 (1987).

"T. R. Dyke, B. J. Howard, and W. Klemperer, J. Chem. Phys. 56, 2442 (1972).

12 J. T. Hougen and N. Ohashi, J. Mol. Spectrosc. 109, 134 (1985).

${ }^{13}$ G. A. Blake, K. L. Busarow, R. C. Cohen, K. B. Laughlin, Y. T. Lee, and R. J. Saykally, J. Chem. Phys. 89, 6577 (1988); G. A. Blake and R. E. Bumgarner, ibid. 91, 7300 (1989).

14 J. A. Odutola, T. A. Hu, D. A. Prinslow, S. E. O'dell, and T. R. Dyke, J. Chem. Phys. 88, 5352 (1988).

${ }^{15}$ J. T. Hougen, J. Mol. Spectrosc. 114, 395 (1985); L. H. Coudert and J. T. Hougen, ibid. 130.86 (1988).

${ }^{10}$ M. J. Frisch, J. E. Del Bene, J. S. Binkley, and H. F. Schaefer III, J. Chem. Phys. 84, 2279 (1986); M. J. Frisch, J. A. Pople, and J. E. Del Bene, J. Phys. Chem. 89, 3664 (1985).

${ }^{17}$ Z. Latajka and S. Scheiner, J. Chem. Phys. 84, 341 (1986).

${ }^{18}$ S-Y. Liu, C. E. Dykstra, K. Kolenbrander, and J. M. Lisy, J. Chem. Phys. 85, 2077 (1986); C. E. Dykstra and L. Andrews, ibid. 92, 6043 (1990).

${ }^{19}$ K. P. Sagarik, R. Ahlrichs, and S. Brode, Mol. Phys. 57, 1247 (1986).

${ }^{20}$ D. M. Hassett, C. J. Marsden, and B. J. Smith, Chem. Phys. Lett. 183, 449 (1991).

${ }^{21}$ M. Havenith, R. C. Cohen, K. L. Busarow, D.-H. Gwo, Y. T. Lee, and R. J. Saykally, J. Chem. Phys. 94, 4476 (1991).

${ }^{22}$ J. W. I. van Bladel, A. van der Avoird, P. E. S. Wormer, and R. J. Saykally, J. Chem. Phys. 97, 4750 (1992), following paper.

${ }^{23} \mathrm{E}$. Zwart, Ph.D. thesis, University of Nijmegen, 1991 (unpublished).

${ }^{24}$ G. A. Blake, K. B. Laughlin, R. C. Cohen, K. L. Busarow, D-H. Gwo, C. A. Schmuttenmaer, D. W. Steyert, and R. J. Saykally, Rev. Sci. Instrum. 62, 1701 (1991).

${ }^{25}$ D. Kaur, A. M. de Souze, J. Wanna, S. A. Hammad, L. Mercorelli, and
D. S. Perry, Appl. Opt. 29, 119 (1990). We use a modified version of this multipass cell in which the input and output beams pass to and from the cell from the same side.

${ }^{26}$ K. L. Busarow, G. A. Blake, K. B. Laughlin, R. C. Cohen, Y. T. Lee, and R. J. Saykally, J. Chem. Phys. 89, 1268 (1988). The similar earlier $5 \mathrm{~cm}$ version of the slit nozzle is described in this paper.

${ }^{27}$ The measured rest frequencies of these far-infrared laser lines are listed in M. Ignuscio, G. Moruzzi, K. M. Evenson, and D. A. Jennings, J. Appl. Phys. 60, R161 (1986), except for the 850 and $939 \mathrm{GHz}$ laser lines, which were measured in this laboratory.

${ }^{28}$ R. E. Bumgarner and G. A. Blake, Chem. Phys. Lett. 161, 308 (1989),

${ }^{29}$ E. Zwart, H. Linnartz, W. L. Meerts, G. T. Fraser, D. D. Nelson, and W. Klemperer, J. Chem. Phys. 95, 793 (1991).

${ }^{30}$ C. H. Townes and A. L. Schawlow, Microwave Spectroscopy (Dover, New York, 1975)

${ }^{31}$ The complete list of all the observed transitions may be obtained from PAPS. See AIP document No. PAPS JCPSA-97-4727-19 for 19 pages of supplemental material. Order by PAPS number and journal reference from American Institute of Physics, Physics Auxiliary Publication Service, 335 East 45th Street, New York, NY 10017. The price is $\$ 1.50$ for each microfiche (60 pages) or $\$ 5.00$ for photocopies of up to 30 pages, and $\$ .015$ for each additional page over 30 pages. Airmail additional. Make checks payable to the American Institute of Physics.

${ }^{32}$ G. Brocks, A. van der Avoird, B. T. Sutciffe, and J. Tennyson, Mol. Phys. 50, 1025 (1983)

${ }^{33}$ J. A. Odutola, T. R. Dyke, B. J. Howard, and J. S. Muenter, J. Chem Phys. 70, 4884 (1979).

${ }^{34}$ P. A. Stockman, R. E. Bumgarner, S. Suzuki, and G. A. Blake, J. Chem. Phys. 96, 2496 (1992).

${ }^{35}$ L. H. Coudert and J. T. Hougen, J. Mol. Spectrose. 149, 73 (1991).

${ }^{36}$ J. W. I. van Bladel, A. van der Avoird, and P. E. S. Wormer, J. Phys. Chem. 95, 5414 (1991).

${ }^{37}$ C. A. Schmuttenmaer, Ph.D. thesis, University of California at Berkeley, 1991 (unpublished).

${ }^{38}$ G. T. Fraser, D. D. Nelson, A. Charo, and W. Klemperer, J. Chem. Phys. 82, 2535 (1985).

${ }^{39}$ D. M. Hassett, C. J. Marsden, and B. J. Smith (private communication).

${ }^{40}$ F. Huisken and T. Pertsch, Chem. Phys. 126, 213 (1988).

${ }^{41}$ M. Snels, R. Fantoni, R. Sanders, and W. L. Meerts, Chem. Phys. 115, 79 (1987).

${ }^{42}$ P. R. Bunker, V. C. Epa, P. Jensen, and A. Karpfen, J. Mol. Spectrosc. 146, 200 (1991).

${ }^{43}$ W. J. Lafferty, R. D. Suenram, and F. J. Lovas, J. Mol. Spectrosc. 123, 434 (1987).

${ }^{44}$ A. E. Barton and B. J. Howard, Discuss. Faraday Soc. 73, 45 (1982).

${ }^{45}$ B. J. Smith, D. J. Swanton, J. A. Pople, H. F. Schaefer III, and L. Radom, J. Chem. Phys. 92, 1240 (1990).

${ }^{46}$ G. Herzberg, Spectra of Diatomic Molecules (van Nostrand, New York, 1950).

${ }^{47}$ R. C. Cohen and R. J. Saykally, J. Phys. Chem. 94, 7991 (1990). 